DESY 05-002

hep-ph/0501178

$\mathrm{SFB} / \mathrm{CPP}-05-02$

January 2005

\title{
Mellin Moments of the Next-to-next-to Leading Order Coefficient Functions for the Drell-Yan Process and Hadronic Higgs-Boson Production
}

\author{
Johannes Blümlein $^{a}$ and Vajravelu Ravindran ${ }^{a, b}$ \\ a Deutsches Elektronen-Synchrotron, DESY, \\ Platanenallee 6, D-15738 Zeuthen, Germany \\ ${ }^{b}$ Harish-Chandra Research Institute, Chhatnag Road, \\ Jhunsi, Allahabad, India.
}

\begin{abstract}
We calculate the Mellin moments of the next-to-next-to leading order coefficient functions for the Drell-Yan and Higgs production cross sections. The results can be expressed in terms of multiple finite harmonic sums of maximal weight $w=4$. Using algebraic and structural relations between harmonic sums one finds that besides the single harmonic sums only five basic sums and their derivatives w.r.t. the summation index contribute. This representation reduces the large complexity being present in $x$-space calculations and is well suited for fast numerical implementations.
\end{abstract}




\section{Introduction}

The Principle of Simplicity ${ }^{1}$ is one of the guiding principles in physics [1]. Whenever possible one seeks for as simple as possible expressions, not only to obtain the result in a more compact form, but also to reveal the basic structures behind. This applies also to complex computations in particle physics. Without achieving suitable simplifications it is often impossible to undertake even more involved calculations, such as one order higher in the coupling constant in perturbation theory.

In the present paper we seek for a simplification of the 2-loop coefficient functions for the Drell-Yan (DY) process both for unpolarized and polarized nucleons, and associated to it, to those for hadronic scalar and pseudo-scalar Higgs boson production in the heavy-mass limit. ${ }^{2}$ The pioneering 2-loop calculations ${ }^{3}$ in this field [5,6] were carried out during the last 15 years and paved the way to understand single-scale quantities in Quantum Chromodynamics (QCD), beyond the level of the pole terms. They form one of the milestone in the history of QCD. The calculations were performed in $x$-space using the QCD-improved parton model. Here $x$ denotes the fraction of the momentum of a radiated particle to that of the source particle. For the representation of the Wilson coefficients a set of up to 77 functions is needed, not counting those, which lead structurally to the same Mellin transform [7].

With the present collider Tevatron [8] and the upcoming large hadron collider, LHC [9], at CERN, the need for precise predictions from theory has become more and more important. These experiments are aimed at not only to discover new particles but also to measure various parameters of the Standard Model at higher precision to confirm the predictions of the theory. The Higgs boson of the Standard Model has yet to be discovered at these machines. Needless to mention that one also hopes to discover various new particles predicted in scenarios beyond the Standard Model, as for example supersymmetric particles or leptoquarks. The measurement of the masses and couplings of newly discovered particles likewise the known particles require the precise knowledge of the QCD corrections.

On the theoretical side the relevant observables have to be calculated at high accuracy. The QCD corrections have a considerable impact both in the discovery channels as well as for the precision measurements. Theoretical uncertainties emerge from ultraviolet (UV) renormalization and mass factorization scale dependence. These uncertainties can only be diminished extending the calculations to higher orders.

In the differential and total production cross sections for the Drell-Yan process as well as for Higgs production at hadron colliders the UV-renormalization scale dependence is due to the strong coupling constant. On the other hand, processes like Higgs production are associated to heavy quark final states such as $t \bar{t}, b \bar{b}$, or inclusive heavy quark productions. They receive a UV-renormalization scale dependence also from heavy quark mass effects.

A second source of uncertainty is due to mass factorization. Both the partonic cross sections and the non-perturbative parton densities depend on the factorization scale. Since the partonic cross sections are computable order by order in perturbation theory, their dependence on the factorization scale is known completely. Similarly, one can fully determine the factorization scale dependence of the parton densities through solving the associated renormalization group equations (RGE) which govern their evolution. To diminish both effects significantly usually

\footnotetext{
${ }^{1}$ PluRAlitas nON EST PONENDA SINE NECCESITATE.

${ }^{2}$ For the calculation of the Higgs and pseudo-scalar Higgs production cross section including mass effects to NLO see [2] and the recent reviews [3].

${ }^{3}$ For NLO results in the case of Higgs-production see [4].
} 
next-to-next-to leading order (NNLO) QCD-corrections have to be performed.

In recent years, there have been several significant developments in achieving very precise theoretical results to match with the accuracy of the experiments. With the advent of the NNLO non-singlet and singlet anomalous dimensions [10] NNLO predictions for the Drell-Yan [5] and Higgs-production cross sections [6] are possible. To get some feeling for the theoretical uncertainty coming from the scale-dependence we consider the following two processes: the total Higgs production cross section and the cross section for Higgs production associated with a top anti-top quark pair. The total Higgs production cross section is one of the most important processes at LHC from the discovery point of view. This production cross section receives dominant contributions from gluon-gluon fusion through the top quark loop. Since the leading order correction is of $O\left(\alpha_{s}^{2}\right)$ and also involves two gluon densities in the initial state, the NLO correction is inevitable to reduce the scale uncertainty. Unfortunately the NLO correction is about $80-100 \%$ larger than the LO correction, casting doubt on the reliability of perturbation theory. Interestingly, the NNLO corrections to the process not only give smaller contributions but also reduce the scale uncertainty significantly, which improves the stability of the perturbative result. Similarly, Higgs production associated with a top anti-top pair suffers from huge scale uncertainties of the order of $100-200 \%$ at the LO level. The NLO correction stabilizes the result significantly. The Drell-Yan cross section, which is known upto NNLO [5], not only tests the reliability of perturbative QCD but also reduces the uncertainties coming from theory in order to make background studies more reliable for new particle searches and physics beyond the Standard Model.

The NLO and NNLO results are technically complicated and they result in large expressions involving a large number of functions. For example the perturbativly computable coefficient functions at NNLO for the Drell-Yan or Higgs total cross section depend on nearly 80 individual functions. These belong to the class of Nielsen integrals [11] with a variety of partly complicated arguments and products with logarithms or the weights $1 /(1 \pm x)$. For the physical cross sections these are convoluted with the respective evolved NNLO parton densities. Since the different sets of parton density are usually available in form of numerical codes, the Mellin convolution is done performing the respective integrals numerically. Because of the complexity of the expressions involved, the numerical computations are usually slow.

We will discuss here an alternative method which is faster to compute the cross sections. It involves the use of the Mellin transformation technique both for the Wilson coefficients and the parton densities. The evolution equations of the parton densities can be solved analytically in Mellin-space. If the respective Wilson coefficients are also known in Mellin-space one may calculate the cross sections by a single numerical integral around the singularities of the product of both in the complex plane. This requires the Mellin-representation of the Wilson coefficients in the variable $N$ and their analytic continuation to complex values of $N$ [12]. ${ }^{4}$ For integer values of $N$ the Mellin transformation of the Wilson coefficients leads to finite harmonic sums $[7,14]$. We use various algebraic relations between these sums $[7,15-17]$ to simplify the expressions. Furthermore, structural relations between the Mellin transforms [18] are used to reduce the number of basic functions further. The use of finite harmonic leads to a synchronization of the expressions. Several complicated harmonic sums present for individual contributions disappear in the final result. A brief summary of the results presented below was given in [19]. Similar investigations as performed in the present paper are carried out for the Wilson coefficients for deeply inelastic scattering off unpolarized and polarized targets [20] and the unpolarized and

\footnotetext{
${ }^{4}$ The analytic continuations of the basic Mellin transforms which contribute to the 3-loop anomalous dimensions have been given in [13] recently.
} 
polarized time-like fragmentation functions [21] to NNLO.

The paper is organized as follows. In section 2 we summarize aspects of the calculation of $2-$ loop Wilson coefficients in $x$-space. The Mellin moments are discussed in section 3 . In section 4 a brief survey is given on multiple harmonic sums and their relation to Mellin transforms. The algebraic relations between the finite harmonic sums used in the present paper is discussed in section 5. In section 6 the basic functions representing the 2-loop Wilson coefficients for the processes considered in the present paper are identified. In the appendices A.1,2 the explicit results are summarized for the Mellin transforms of the NNLO Wilson coefficients for the unpolarized and polarized Drell-Yan process and appendix A.3,4 contains the corresponding expressions for the hadronic scalar and pseudo-scalar Higgs boson production processes.

\section{Coefficient Functions}

Due to mass factorization, hadronic cross sections such as those for the Drell-Yan process [5] and hadronic Higgs boson production [6] can be expressed in terms of Mellin convolutions of the perturbativly computable coefficient functions $\Delta_{a b}\left(x, Q^{2}, \mu^{2}\right)$ and non-perturbative parton distributions, $f_{a}\left(x, \mu^{2}\right)$, of incoming hadrons,

$$
\sigma\left(x, Q^{2}\right)=\int_{0}^{1} d x_{1} \int_{0}^{1} d x_{2} \int_{0}^{1} d z f_{a}\left(x_{1}, \mu^{2}\right) f_{b}\left(x_{2}, \mu^{2}\right) \Delta_{a b}\left(z, \frac{Q^{2}}{\mu^{2}}\right) \delta\left(x-z x_{1} x_{2}\right) .
$$

Here the sum over $a, b=q, \bar{q}, g$ is implied. $Q^{2}$ is the mass squared of the Drell-Yan pair or the Higgs boson. The parameter $x$ is a scaling variable defined by

$$
x=\frac{Q^{2}}{S}, \quad S=\left(P_{1}+P_{2}\right)^{2},
$$

where $P_{1}, P_{2}$ are the momenta of incoming hadrons. We have set both the UV- and factorization scales to be equal to $\mu^{2}$. Though the parton densities are not calculable in the perturbative QCD, their evolution with respect to the factorization scale $\mu^{2}$ is computable using the renormalization group equations (RGE). Hence higher order corrections to hadronic reactions enter through two sources viz, the coefficient functions and the RG equations of the parton distribution functions.

The coefficient functions are computed from the partonic cross sections in powers of the strong coupling constant $\alpha_{s}\left(\mu^{2}\right)$ in perturbative QCD. They are expressible in terms of the scaling variable $z=Q^{2} / s$, where $s$ is the center of mass of incoming partonic system. $Q^{2}$ is a large invariant mass characteristic for the process. The lowest order contributions are of $O\left(\alpha_{s}^{0}\right)$ and the Wilson coefficients are obtained from the Born-diagrams. In the case of the DrellYan process the lowest order contributions are due to quark anti-quark annihilation, likewise gluon-gluon fusion in the case of Higgs boson production through the top-quark loop. Hence the results are just proportional to $\delta(1-z)$. To the next-to-leading order (NLO) one encounters virtual corrections as well as real gluon emissions to the Born-processes. In addition, gluoninitiated processes also contribute in case of the Drell-Yan process as quark-initiated processes in case of Higgs boson production. The virtual processes are in general UV-divergent due to the loop-corrections. Since one is dealing with light partons inside the incoming hadrons, the real emission processes suffer from collinear divergences. In addition, the standard soft divergences appear in both real emission as well as virtual contributions to Born processes. All these divergences are regularized using $n$-dimensional regularization. The UV-divergences are removed by standard UV-renormalization. This introduces the renormalization scale $\mu_{R}^{2}$ 
both through $\alpha_{s}\left(\mu_{R}^{2}\right)$ and $\ln \left(Q^{2} / \mu_{R}^{2}\right)$-terms. The soft divergences cancel when virtual and real emission contributions are added. The remaining collinear divergences are removed by the mass factorization procedure. This introduces a new scale called factorization scale $\mu_{F}^{2}$. Finally one ends up with the regular functions as $\ln (x), \ln (1-x)$, polynomials in $x$ and distribution functions such as $\left(\ln ^{m}(1-x) /(1-x)\right)_{+}$with $m=0,1 ; \delta(1-x)$.

Beyond NLO, the computation becomes more complicated. First of all there are many more processes that contribute to this order. For example one finds, two-loop virtual contributions to the Born terms, one loop virtual corrections to the NLO contributions, double real emissions to the Born contributions, and single real emissions to the NLO processes. In addition several new type of processes start contributing from NNLO level onwards. Due to this, the number of integrations involved increases enormously and new functions emerge. The two-loop virtual corrections to the Born process generate only constants such as $\zeta_{2}, \zeta_{3}, \zeta_{4}$. But one-loop corrections to NLO processes and real emissions to Born-, NLO- and the new NNLO-processes lead to hypergeometric functions $F_{2,1}$ which upon integration generate further higher functions. The hypergeometric functions usually result from the one-loop box to NLO processes and also from angular integration of the real emission processes at the NNLO level. Higher functions are also generated when NLO-splitting functions are convoluted with the Born- and NLO-cross sections. Such convolutions are required in order to perform mass factorization. The NNLO results are finally expressible in terms of polynomials in $x$, logarithms and Nielsen integrals $S_{n, p}(x)$ [11], both with various argument-functions, defined by

$$
S_{n, p}(x)=\frac{(-1)^{n+p-1}}{(n-1) ! p !} \int_{0}^{1} \frac{d z}{z} \ln ^{n-1}(z) \ln ^{p}(1-z x)
$$

The weight $w$ of these functions is defined by $w=p+n$, where any power of a logarithm counts for $w=1$ as the case for the denominators $1 / x, 1 /(1+x)$ and $1 /(1-x)$. In a product of functions the weights of the factors add. The standard polylogarithms are related to the Nielsen integrals by :

$$
\begin{aligned}
\operatorname{Li}_{n}(x) & =\frac{d \operatorname{Li}_{n+1}(x)}{d \ln (x)} \equiv S_{n-1,1}(x) \\
& =\frac{(-1)^{n-1}}{(n-2)} \int_{0}^{1} \frac{d z}{z} \ln ^{n-2}(z) \ln (1-z x) \quad \text { for } \quad n \geq 2 .
\end{aligned}
$$

One relates the logarithms to the polylogarithms by simple differentiation:

$$
\begin{aligned}
\frac{d \operatorname{Li}_{2}( \pm x)}{d \ln (x)} & =\operatorname{Li}_{1}( \pm x)=-\ln (1 \mp x) \\
\operatorname{Li}_{0}(x) & =\frac{x}{1-x}
\end{aligned}
$$

Similarly, one reduces the weight of a Nielsen integral by differentiating w.r.t. $\ln (x)$ :

$$
\frac{d S_{n, p}(x)}{d \ln (x)}=S_{n-1, p}(x) .
$$

In addition, distributions as $\delta(1-x)$ and $\left(\ln ^{m}(1-x) /(1-x)\right)_{+}$with $m=0,1,2,3$ contribute. These functions and their Mellin transforms can be found in Ref. [7]. 
The hadronic scattering cross sections are obtained using Eq. (1). One performs the integration over $x_{1}$ and $x_{2}$ after folding the perturbativly computed coefficient functions with the appropriate parton distributions. This may involve further evaluation of various Nielsen integrals and increases the complexity of the numerical evaluation of the hadronic cross sections. In the next section we will study the structure of these corrections in Mellin space using algebraic identities which relate the resulting finite harmonic sums. We will present an alternative treatment of the evaluation of the total cross sections upto NNLO by working in Mellin space. Such techniques have been used in the past to compute deep-inelastic scattering cross sections. They are also found to be most suitable for various resummation programs [22]. ${ }^{5}$

\section{Mellin Moments}

The Mellin-transform [24] of a given function $F(x)$ is defined by

$$
\mathbf{M}[F](N)=\int_{0}^{1} d x x^{N-1} F(x) .
$$

The Mellin convolution of two functions $F_{1}(x), F_{2}(x)$ is given by

$$
\left[F_{1} \otimes F_{2}\right](x)=\int_{0}^{1} d x_{1} \int_{0}^{1} d x_{2} F_{1}\left(x_{1}\right) F_{2}\left(x_{2}\right) \delta\left(x-x_{1} x_{2}\right)
$$

$\mathbf{M}\left[\left[F_{1} \otimes F_{2}\right](x)\right](N)$ reduces to the product of Mellin moments of $F_{1}(x)$ and $F_{2}(x)$, i.e.

$$
\mathbf{M}\left[F_{1} \otimes F_{2}\right](N)=\mathbf{M}\left[F_{1}\right](N) \cdot \mathbf{M}\left[F_{2}\right](N)
$$

One may generalize this property for the convolution of $m$ functions :

$$
\begin{gathered}
{\left[F_{1} \otimes F_{2} \otimes \ldots \otimes F_{m}\right](x)=\int_{0}^{1} d x_{1} \int_{0}^{1} d x_{2} \ldots \int_{0}^{1} d x_{m} \int_{0}^{1} d z F_{1}\left(x_{1}\right) F_{2}\left(x_{2}\right) \ldots F_{m}\left(x_{m}\right)} \\
\times \delta\left(x-x_{1} x_{2} \ldots x_{m}\right) \\
\mathbf{M}\left[F_{1} \otimes F_{2} \otimes \ldots F_{m}\right](N)=\prod_{k=1}^{m} \mathbf{M}\left[F_{k}\right](N) .
\end{gathered}
$$

Indeed, already the multiple convolution of rather simple functions in $x$-space may lead to complicated expressions, cf. Ref. [25]. Contrary to that, the representation (11) is straightforward.

Due to mass-factorization the QCD-improved collinear parton model relates the hadronic cross sections via Mellin convolutions to the partonic cross sections and the parton distribution functions. Hence the cross section in the Mellin- $N$ space becomes

$$
\mathbf{M}[\sigma]\left(N, Q^{2}\right)=\mathbf{M}\left[f_{a}\right]\left(N, \mu^{2}\right) \mathbf{M}\left[f_{b}\right]\left(N, \mu^{2}\right) \mathbf{M}\left[\Delta_{a b}\right]\left(N, \frac{Q^{2}}{\mu^{2}}\right) .
$$

The Mellin moments of these functions can be analytically continued [12] to complex values of $N$ so that one can use various analyticity properties of these functions in complex $N$-space to evaluate them efficiently. To retrieve back the full $x$-dependent result for (12), we have to take the inverse Mellin transform as a numerical contour integral around all singularities in $N$.

\footnotetext{
${ }^{5}$ Heavy flavor contributions in Mellin space were treated in [23].
} 
For our analysis, the starting point is Eq. (8) with given parton densities $f_{a}\left(x, \mu^{2}\right)$ and known coefficient functions $\Delta_{a b}\left(x, Q^{2}\right)$ computed upto NNLO in perturbative QCD. We then compute the Mellin moments of these functions in $N$-space and analytically continue them to complex $N$-space. At the end, we use Eq. (12) and perform the inverse Mellin transformation to arrive at the results in $x$-space using a suitable contour in the complex $N$-space. Since only one integral has to be carried out numerically, the evaluation can be performed very fast.

Before we study the Mellin moment of the coefficient functions, we would like to make a few remarks on the parton densities. As is well known, the parton densities are fitted as functions of $x$ using the available deeply inelastic scattering data. The parton distributions are determined at some scale $Q_{0}^{2}$ along with the QCD-scale, $\Lambda_{\mathrm{QCD}}$. The renormalization group equations for mass factorization in $x$-space

$$
\mu^{2} \frac{d f_{a / P}\left(x, \mu^{2}\right)}{d \mu^{2}}=\frac{\alpha_{s}\left(\mu^{2}\right)}{4 \pi} \sum_{b=q, \bar{q}, g} \int_{x}^{1} \frac{d z}{z} P_{a b}\left(z, \alpha_{s}\left(\mu^{2}\right)\right) f_{b / P}\left(z, \mu^{2}\right) \quad a=q, \bar{q}, g
$$

relate the parton densities at $Q_{0}^{2}$ to those at the scale $\mu^{2}$. The splitting functions $P_{a b}\left(z, \alpha_{s}\left(\mu^{2}\right)\right)$ are computable order by order in perturbation theory:

$$
P_{a b}\left(z, \alpha_{s}\left(\mu^{2}\right)\right)=\sum_{n=0}^{\infty}\left(\frac{\alpha_{s}\left(\mu^{2}\right)}{4 \pi}\right)^{n} P_{a b}^{(n)}(z)
$$

Instead solving the integro-differential equations (13) one may Mellin-transform these equations to

$$
\mu^{2} \frac{d \mathbf{M}\left[f_{a / P}\right]\left(N, \mu^{2}\right)}{d \mu^{2}}=\frac{\alpha_{s}\left(\mu^{2}\right)}{4 \pi} \sum_{b=q, \bar{q}, g} \mathbf{M}\left[P_{a b}\right]\left(N, \alpha_{s}\left(\mu^{2}\right)\right) \mathbf{M}\left[f_{b / P}\right]\left(N, \mu^{2}\right) .
$$

The solution of (15) for $\mathbf{M}\left[f_{a / P}\right]\left(N, \mu^{2}\right)$ is straightforward as it is now just a first order differential equation. The parton densities in $x$-space are obtained from the solutions $\mathbf{M}\left[f_{a / P}\right]\left(N, \mu^{2}\right)$ by an inverse Mellin transformation. Since we are dealing with cross sections in $N$-space given in (12), the solutions $\mathbf{M}\left[f_{a / P}\right]\left(N, \mu^{2}\right)$ can be used for further analysis directly.

The next task is to compute the Mellin moments of the known coefficient functions for the different hard processes in case of massless fermions to 2-loop order which were usually computed in $x$-space.

Let us start with the Drell-Yan process. We present here the relevant formulae for both unpolarized as well as polarized cross sections. The Drell-Yan process is given by

$$
H_{1}\left(P_{1}\right)+H_{2}\left(P_{2}\right) \rightarrow l^{+}\left(k_{1}\right)+l^{-}\left(k_{2}\right)+X,
$$

where $H_{i}$ are the incoming hadrons with momenta $P_{i} . k_{i}$, with $i=1,2$, are the momenta of final state leptons $l^{+} l^{-}$, respectively. Since the dominant contribution is through the $\gamma$-exchange $s$-channel processes ${ }^{6}$, we find the cross section can be expressed as

$$
\frac{d(\Delta) \sigma^{D Y}\left(x, Q^{2}\right)}{d Q^{2}}=\frac{4 \pi \alpha^{2}}{3 N_{c} Q^{2} S}(\Delta) W^{D Y}\left(x, Q^{2}\right)
$$

where $(\Delta)$ denotes the polarized case, $Q^{2}$ is the invariant mass of the di-lepton,

$$
S=\left(P_{1}+P_{2}\right)^{2}, \quad x=\frac{Q^{2}}{S},
$$

\footnotetext{
${ }^{6}$ The $Z$-exchange processes can be incorporated in a similar way.
} 
$N_{c}$ is the number of colors, and $\alpha$ is the electromagnetic coupling constant. The scaling function, usually called the hadronic Drell-Yan structure functions $(\Delta) W^{D Y}\left(x, Q^{2}\right)$, is related to the coefficient functions as

$$
\begin{aligned}
(\Delta) W^{D Y}\left(x, Q^{2}\right)= & \sum_{a, b=q, \bar{q}, g} \int_{0}^{1} d x_{1} \int_{0}^{1} d x_{2} \int_{0}^{1} d z(\Delta) f_{a / H_{1}}\left(x_{1}, \mu^{2}\right)(\Delta) f_{b / H_{2}}\left(x_{2}, \mu^{2}\right) \\
& \times(\Delta) \Delta_{a b}^{D Y}\left(z, Q^{2}, \mu^{2}\right) \delta\left(x-z x_{1} x_{2}\right)
\end{aligned}
$$

where we have set the renormalization scale to be equal to the factorization scale. Alternatively, we can express the above equation as

$$
(\Delta) W^{D Y}\left(x, Q^{2}\right)=\sum_{a, b=q, \bar{q}, g} \int_{x}^{1} \frac{d y}{y}(\Delta) \Phi_{a b}\left(y, \mu^{2}\right)(\Delta) \Delta_{a b}^{D Y}\left(\frac{x}{y}, Q^{2}, \mu^{2}\right),
$$

where the flux $\Phi_{a b}$ is defined by

$$
(\Delta) \Phi_{a b}\left(y, \mu^{2}\right)=\int_{y}^{1} \frac{d z}{z}(\Delta) f_{a / H_{1}}\left(z, \mu^{2}\right)(\Delta) f_{b / H_{2}}\left(\frac{y}{z}, \mu^{2}\right) .
$$

The Mellin moment of $x^{-1}\left[d(\Delta) \sigma^{D Y}\left(x, Q^{2}\right) / d Q^{2}\right]$ reduces to

$$
\mathbf{M}\left[\frac{1}{x} \frac{d(\Delta) \sigma^{D Y}}{d Q^{2}}\right]\left(N, \mu^{2}\right)=\frac{4 \pi \alpha^{2}}{3 N_{c} Q^{4}} \mathbf{M}\left[(\Delta) W^{D Y}\right]\left(N, \mu^{2}\right),
$$

where

$$
\mathbf{M}\left[(\Delta) W^{D Y}\right]\left(N, \mu^{2}\right)=\mathbf{M}\left[(\Delta) \Phi_{a b}\right]\left(N, \mu^{2}\right) \mathbf{M}\left[(\Delta) \Delta_{a b}^{D Y}\right]\left(N, \mu^{2}\right) .
$$

The flux $\mathbf{M}\left[(\Delta) \Phi_{a b}\right]\left(N, \mu^{2}\right)$ can be computed using (15) with the input parton densities as boundary conditions.

We now present the total cross section for both scalar $(H)$ and pseudo-scalar $(A)$ Higgs bosons at hadron colliders. The process is given by

$$
H_{1}\left(P_{1}\right)+H_{2}\left(P_{2}\right) \rightarrow B+X
$$

where $B=H, A$. The total cross section for Higgs boson production is found to be

$$
\begin{array}{r}
\sigma_{\text {tot }}^{B}\left(x, m^{2}\right)=\frac{\pi G_{B}^{2}}{8\left(N_{c}^{2}-1\right)} \sum_{a, b=q, \bar{q}, g} \int_{x}^{1} d x_{1} \int_{\frac{x}{x_{1}}}^{1} d x_{2} f_{a / H_{1}}\left(x_{1}, \mu^{2}\right) f_{b / H_{2}}\left(x_{2}, \mu^{2}\right) \\
\times \Delta_{a b, B}\left(\frac{x}{x_{1} x_{2}}, m^{2}, \mu^{2}\right), \quad B=H, A,
\end{array}
$$

where $m^{2}$ is the mass of the Higgs boson and the scaling variable is defined by

$$
x=\frac{m^{2}}{S}, \quad S=\left(P_{1}+P_{2}\right)^{2} .
$$

$\Delta_{a b, B}\left(x, m^{2}, \mu^{2}\right)$ is the partonic coefficient function. Again we have set both renormalization and factorization scale to be equal. The overall constant $G_{B}$ is

$$
G_{B}=-2^{5 / 4} a_{s}\left(\mu_{R}^{2}\right) G_{F}^{2} \tau_{B} F_{B}\left(\tau_{B}\right) \mathcal{C}_{B}\left(a_{s}\left(\mu_{R}^{2}\right), \mu_{R}^{2}, m_{t}^{2}\right)
$$


with

$$
a_{s}\left(\mu_{R}^{2}\right)=\frac{\alpha_{s}\left(\mu_{R}^{2}\right)}{4 \pi},
$$

where $\mu_{R}$ is the renormalization scale. $G_{F}$ is the Fermi constant and the functions $F_{B}(\tau)$ are given by

$$
\begin{aligned}
F_{H}(\tau) & =1+(1-\tau) f(\tau), \quad F_{A}(\tau)=f(\tau) \cot \beta \\
\tau & =\frac{4 m_{t}^{2}}{m^{2}} \\
f(\tau) & =\arcsin ^{2} \frac{1}{\sqrt{\tau}}, \quad \text { for } \quad \tau \geq 1, \\
f(\tau) & =-\frac{1}{4}\left(\ln \frac{1-\sqrt{1-\tau}}{1+\sqrt{1-\tau}}+\pi i\right)^{2} \quad \tau \leq 1,
\end{aligned}
$$

where $\beta$ is the mixing angle in the two-Higgs doublet model. $m_{t}$ is the mass of the top quark. The coefficient $\mathcal{C}_{B}$ is given by

$$
\begin{aligned}
\mathcal{C}_{H}\left(a_{s}\left(\mu_{R}^{2}\right), m_{t}^{2}\right)= & 1+a_{s}^{(5)}\left(\mu_{R}^{2}\right)\left[5 C_{A}-3 C_{F}\right]+\left(a_{s}^{(5)}\left(\mu_{R}^{2}\right)\right)^{2}\left[\frac{27}{2} C_{F}^{2}\right. \\
& -\frac{100}{3} C_{A} C_{F}+\frac{1063}{36} C_{A}^{2}-\frac{4}{3} C_{F} T_{f}-\frac{5}{6} C_{A} T_{f}+\left(7 C_{A}^{2}-11 C_{A} C_{F}\right) \ln \frac{\mu_{R}^{2}}{m_{t}^{2}} \\
& \left.+n_{f} T_{f}\left(-4 C_{F}-\frac{47}{9} C_{A}+8 C_{F} \ln \frac{\mu_{R}^{2}}{m_{t}^{2}}\right)\right], \\
\mathcal{C}_{A}\left(a_{s}\left(\mu_{R}^{2}\right), m_{t}^{2}\right)= & 1,
\end{aligned}
$$

[26], where $a_{s}^{(5)}$ refers to the five-flavor number scheme. The color factors are

$$
C_{A}=N_{c}, \quad C_{F}=\frac{N_{c}^{2}-1}{2}, \quad T_{f}=\frac{1}{2} .
$$

Eq. (25) can be expressed in a compact form as

$$
\frac{1}{x} \sigma_{t o t}^{B}\left(x, m^{2}\right)=\frac{\pi G_{B}^{2}}{8\left(N^{2}-1\right)} \sum_{a, b=q, \bar{q}, g} \int_{x}^{1} \frac{d y}{y} \Phi_{a b}\left(y, \mu^{2}\right) \frac{y}{x} \Delta_{a b, B}\left(\frac{x}{y}, m^{2}, \mu^{2}\right) .
$$

The Mellin transform of (32) becomes

$$
\mathbf{M}\left[\sigma_{t o t}^{B}\right]\left(N, m^{2}\right)=\frac{\pi G_{B}^{2}}{8\left(N^{2}-1\right)} \sum_{a b=q, \bar{q}, g} \mathbf{M}\left[\Phi_{a b}\right]\left(N+1, \mu^{2}\right) \mathbf{M}\left[\Delta_{a b, B}\right]\left(N, m^{2}, \mu^{2}\right) .
$$

The flux $\mathbf{M}\left[\Phi_{a b}\right]$ can be extracted from the solution of the evolution equations (12) and $\mathbf{M}\left[\Delta_{a b, B}\right]$ can be computed analytically from the known functions $\Delta_{a b, B}\left(x, m^{2}, \mu^{2}\right)$, see appendix 3,4 . 


\section{Finite Harmonic Sums}

We are dealing with the total scattering cross sections for the unpolarized and polarized DrellYan process and hadronic (pseudo)scalar Higgs boson production in the heavy-mass limit, which depend only on two variables, $x=Q^{2} / s$ and $Q^{2}$, the invariant mass squared of the final state. The coefficient functions upto NNLO contain a large class of $x$-space functions [7]. Their complexity reaches nearly 80 , the number of all alternating and non-alternating finite harmonic sums $[7$, $17,18]$ of weight $w \leq 4$. The class contains simple functions of the form $(1 \pm x)^{-1}, \ln ^{m}(x)$, $\ln ^{m}(1-x)$, and $\left(\ln ^{m}(1-x) /(1-x)\right)_{+}, m=0 \ldots 3$. More complicated examples are weighted Nielsen-integrals $S_{n, p}(x) /(1 \mp x)$. The Mellin transforms of these functions were evaluated in [7]. In the following we present some examples.

The Mellin moment of the function $1 /(1+x)$ can be computed as follows:

$$
\begin{aligned}
\int_{0}^{1} d x x^{N-1} \frac{1}{(1+x)} & =\int_{0}^{1} d x x^{N-1} \sum_{i=0}^{\infty}(-1)^{i} x^{i}=\sum_{i=0}^{\infty} \frac{(-1)^{i}}{i+N} \\
& =(-1)^{N-1}\left[\sum_{i=1}^{N-1} \frac{(-1)^{i}}{i}-\sum_{i=1}^{\infty} \frac{(-1)^{i}}{i}\right]=(-1)^{N-1}\left[S_{-1}(N-1)+\ln (2)\right](34)
\end{aligned}
$$

Likewise one obtains

$$
\begin{aligned}
\int_{0}^{1} d x x^{N-1} \frac{1}{(1-x)_{+}} & =\int_{0}^{1} d x \frac{x^{N-1}-1}{1-x}=\int_{0}^{1} d x\left(x^{N-1}-1\right) \sum_{i=0}^{\infty} x^{i} \\
& =\sum_{i=0}^{\infty}\left(\frac{1}{i+N}-\frac{1}{i+1}\right)=-S_{1}(N-1)
\end{aligned}
$$

where the finite harmonic sums are defined as

$$
S_{k}(N)=\sum_{i=1}^{N} \frac{1}{i^{k}}, \quad S_{-k}(N)=\sum_{i=1}^{N} \frac{(-1)^{k}}{i^{k}} .
$$

Similarly, we compute the Mellin moment of $x^{r} \ln (1-x)$ by expanding

$$
\ln (1-x)=-\sum_{i=1}^{\infty} \frac{x^{i}}{i}
$$

using partial fractions and changing the limits of the sums :

$$
\begin{aligned}
\int_{0}^{1} d x x^{N-1} x^{r} \ln (1-x) & =-\sum_{i=1}^{\infty} \frac{1}{i(i+N+r)}=\frac{1}{N+r} \sum_{i=1}^{\infty}\left(\frac{1}{i+N+r}-\frac{1}{i}\right) \\
& =-\frac{1}{N+r} \sum_{i=1}^{N+r} \frac{1}{i}=-\frac{S_{1}(N+r)}{N+r} .
\end{aligned}
$$

One may compute the Mellin moment of the distribution

$$
\begin{aligned}
\int_{0}^{1} d x x^{N-1}\left(\frac{\ln (1-x)}{1-x}\right)_{+} & =\int_{0}^{1} d x\left[x^{N-1}-1\right] \frac{\ln (1-x)}{1-x}=\frac{N-1}{2} \int_{0}^{1} x^{N-2} \ln ^{2}(1-x) \\
& =\frac{N-1}{2} \sum_{i, j=1}^{\infty} \frac{1}{i j(i+j+N-1)} .
\end{aligned}
$$


The summation is performed after partial fractioning and shifting the summation limits and one arrives at

$$
\int_{0}^{1} d x x^{N-1}\left(\frac{\ln (1-x)}{1-x}\right)_{+}=S_{1,1}(N-1) .
$$

The latter sum is a multiple (nested) harmonic sum defined by

$$
S_{m, m_{1}, m_{2}, \ldots, m_{k}}(N)=\sum_{i=1}^{N} \frac{S_{m_{1}, m_{2}, \ldots, m_{k}}(i)}{i^{m}}, \forall m_{i}>0
$$

In general, also alternating nested harmonic sums contribute, which are labeled with as well negative indices $k_{i}<0$,

$$
S_{k_{1} \ldots k_{m}}(N)=\sum_{n_{1}=1}^{N} \frac{\left[\operatorname{sign}\left(k_{1}\right)\right]^{n_{1}}}{n_{1}^{\left|k_{1}\right|}} \sum_{n_{2}=1}^{n_{1}} \frac{\left[\operatorname{sign}\left(k_{2}\right)\right]^{n_{2}}}{n_{2}^{\left|k_{2}\right|}} \cdots \sum_{n_{m}=1}^{n_{m}-1} \frac{\left[\operatorname{sign}\left(k_{m}\right)\right]^{n_{m}}}{n_{m}^{\left|k_{m}\right|}}
$$

with $k_{l}, l \neq 0$. Similarly one computes Mellin moments of more complicated function. For example,

$$
\begin{aligned}
\mathbf{M}\left[\left(\frac{\ln ^{3}(1-x)}{1-x}\right)_{+}\right](N)= & \frac{1}{4} S_{1}^{4}(N-1)+\frac{3}{2} S_{1}^{2}(N-1) S_{2}(N-1)+\frac{3}{4} S_{2}^{2}(N-1) \\
& +2 S_{1}(N-1) S_{3}(N-1)+\frac{3}{2} S_{4}(N-1)
\end{aligned}
$$

where the right hand side contains only single finite harmonic sums. The Mellin moments of Nielsen integrals can be mostly done by relating them to simpler moments after an integration by parts:

$$
\int_{0}^{1} d x x^{N-1} S_{1,2}(-x)=\frac{1}{N} \frac{\zeta_{3}}{8}-\frac{1}{2 N} \mathbf{M}\left[\ln ^{2}(1+x)\right](N)
$$

The Mellin transforms of individual functions in $x$-space may contain complicated sums [7], which cancel in combinations. As an example we mention

$$
\begin{aligned}
& \int_{0}^{1} d x x^{N-1}\left[S_{1,2}(-x)+\operatorname{Li}_{2}(-x) \ln (1+x)+\frac{1}{2} \ln (x) \ln ^{2}(1+x)\right] \\
& =\frac{(-1)^{N-1}}{N}\left\{S_{-1,2}(N)+\frac{\zeta_{2}}{2}\left[S_{1}(N)-S_{-1}(N)\right]\right\}+\frac{1+(-1)^{N-1}}{N}\left[\frac{\zeta_{3}}{8}-\frac{\ln (2) \zeta_{2}}{2}\right]
\end{aligned}
$$

in which the sum $S_{-1,1}(N)$ does not occur unlike the case for $\mathbf{M}\left[\operatorname{Li}_{2}(-x) \ln (1+x)\right](N)$. We finally mention recursive integral-representations for the finite harmonic sums and weighted power sums, cf. [7],

$$
\begin{aligned}
S_{ \pm k}(N) & =\int_{0}^{1} \frac{d x_{1}}{x_{1}} \cdots \int_{0}^{x_{k-1}} \frac{\left( \pm x_{k}\right)^{N}-1}{x_{k} \mp 1} \\
& =\frac{(-1)^{k-1}}{(k-1) !} \int_{0}^{1} d x \ln ^{k-1}(x) \frac{( \pm x)^{N}-1}{x \mp 1} \\
\sum_{k=1}^{N} \frac{( \pm x)^{k}}{k^{l}} & =\frac{(-1)^{l-1}}{(l-1) !} \int_{0}^{x} d z \ln ^{l-1}(z) \frac{( \pm z)^{N}-1}{z \mp 1} .
\end{aligned}
$$


The translation of the 2-loop Wilson coefficients $[5,6]$ from $x$ - to $N$-space is performed using the tables given in [7] for the individual functions. One observes the cancellation of a series of sums. In particular we would like to mention, that multiple sums with the index $\{-1\}$ do not occur in the final results up to $\mathbf{w}=4$.

\section{Algebraic Relations}

Finite harmonic sums obey algebraic equations, see e.g. [17]. The more simple relations for finite harmonic sums can be found in $[7,15,16,27]$. Finite harmonic sums obey a shuffle algebra. To further simplify the expressions for the Wilson coefficients dealt with in the present paper the following relations are used for sums with two

$$
\begin{aligned}
S_{1,1}(N) & =\frac{1}{2}\left(S_{1}(N)^{2}+S_{2}(N)\right) \\
S_{-1,1}(N) & =-S_{1,-1}(N)+S_{1}(N) S_{-1}(N)+S_{-2}(N) \\
S_{-1,-2}(N) & =-S_{-2,-1}(N)+S_{-2}(N) S_{-1}(N)+S_{3}(N) \\
S_{1,2}(N) & =-S_{2,1}(N)+S_{2}(N) S_{1}(N)+S_{3}(N) \\
S_{-1,2}(N) & =-S_{2,-1}(N)+S_{2}(N) S_{-1}(N)+S_{-3}(N) \\
S_{1,-2}(N) & =-S_{-2,1}(N)+S_{-2}(N) S_{1}(N)+S_{-3}(N) \\
S_{2,2}(N) & =\frac{1}{2}\left(S_{2}(N)^{2}+S_{4}(N)\right) \\
S_{-2,-2}(N) & =\frac{1}{2}\left(S_{-2}(N)^{2}+S_{4}(N)\right) \\
S_{2,-2}(N) & =-S_{-2,2}(N)+S_{-2}(N) S_{2}(N)+S_{-4}(N) \\
S_{-1,-3}(N) & =-S_{-3,-1}(N)+S_{-3}(N) S_{-1}(N)+S_{4}(N) \\
S_{1,3}(N) & =-S_{3,1}(N)+S_{3}(N) S_{1}(N)+S_{4}(N) \\
S_{-1,3}(N) & =-S_{3,-1}(N)+S_{3}(N) S_{-1}(N)+S_{-4}(N) \\
S_{1,-3}(N) & =-S_{-3,1}(N)+S_{-3}(N) S_{1}(N)+S_{-4}(N)
\end{aligned}
$$

and three indices :

$$
\begin{aligned}
S_{1,2,1}(N) & =-2 S_{2,1,1}(N)+S_{3,1}(N)+S_{1}(N) S_{2,1}(N)+S_{2,2}(N) \\
S_{1,1,2}(N) & =S_{2,1,1}(N)+\left[\frac{1}{2}\left(S_{1}(N)\left(S_{1,2}(N)-S_{2,1}(N)\right)+S_{1,3}(N)-S_{3,1}(N)\right]\right. \\
S_{1,-2,1}(N) & =-2 S_{-2,1,1}(N)+S_{-3,1}(N)+S_{1}(N) S_{-2,1}(N)+S_{-2,2}(N) \\
S_{1,1,-2}(N) & =S_{-2,1,1}(N)+S_{-2}(N) S_{2}(N)-S_{-2,2}(N)-S_{-2}(N) S_{1,1}(N)
\end{aligned}
$$




$$
+S_{1}(N) S_{1,-2}(N)+S_{1,-3}(N)-S_{1}(N) S_{-3}(N) .
$$

At intermediate stages of the computation we encounter various more complicated sums such as $S_{1,-1,2}, S_{-1,-1,-2}, S_{-1,-2,-1}, S_{-2,-1,-1}, S_{2,-1,1}, S_{1,2,-1}, S_{2,1-1}, S_{-1,1,2}, S_{-1,1,2}, S_{-1,-3}$, and $S_{-1,3}$. All these sums do finally occur in symmetric combinations, which are polynomials of single harmonic sums. At the level of 3 -fold sums only $S_{-2,1,1}$ and $S_{2,1,1}$ remain.

\section{Coefficient Functions in N-Space}

Let us now express the Wilson coefficients in terms of the remaining harmonic sums in terms of polynomials and as rational functions in $N$. Furthermore, we list the respective harmonic sums in terms of their Mellin-transforms, cf. [7].

The single harmonic sums are :

$$
\begin{aligned}
S_{-4}(N) & =(-1)^{N+1} \frac{1}{6} \mathbf{M}\left[\frac{\ln ^{3}(x)}{1+x}\right](N+1)-\frac{7}{20} \zeta_{2}^{2} \\
S_{-3}(N) & =(-1)^{N} \frac{1}{2} \mathbf{M}\left[\frac{\ln ^{2}(x)}{1+x}\right](N+1)-\frac{3}{4} \zeta_{3} \\
S_{-2}(N) & =(-1)^{N+1} \mathbf{M}\left[\frac{\ln (x)}{1+x}\right](N+1)-\frac{1}{2} \zeta_{2} \\
S_{-1}(N) & =(-1)^{N} \mathbf{M}\left[\frac{1}{1+x}\right](N+1)-\ln (2) \\
S_{4}(N) & =\frac{1}{6} \mathbf{M}\left[\frac{\ln ^{3}(x)}{1-x}\right](N+1)+\frac{2}{5} \zeta_{2}^{2} \\
S_{3}(N) & =-\frac{1}{2} \mathbf{M}\left[\frac{\ln ^{2}(x)}{1-x}\right](N+1)+\zeta_{3} \\
S_{2}(N) & =\mathbf{M}\left[\frac{\ln (x)}{1-x}\right](N+1)+\zeta_{2} \\
S_{1}(N) & =-\mathbf{M}\left[\left(\frac{1}{1-x}\right)+(N+1) .\right.
\end{aligned}
$$

These harmonic sums can be solely expressed in terms of Euler's $\psi$-function and the $\beta$ function [27] and their derivatives, which is related to the former combining two $\psi$-functions with shifted argument. These functions represent at the same time the analytic continuation of these harmonic sums :

$$
\begin{aligned}
S_{k}(N) & =\frac{(-1)^{k+1}}{(k-1) !} \psi^{(k-1)}(N+1)+\zeta(k) \\
S_{-1}(N) & =(-1)^{N} \beta(N+1)-\ln (2) \\
S_{-k}(N) & =\frac{(-1)^{N+k-1}}{(k-1) !} \beta^{(k-1)}(N+1)-\left(1-\frac{1}{2^{k-1}}\right) \zeta_{k}, \quad k \geq 2,
\end{aligned}
$$




$$
\beta(z)=\frac{1}{2}\left[\psi\left(\frac{1+z}{2}\right)-\psi\left(\frac{z}{2}\right)\right]
$$

The following five double sums occur :

$$
\begin{aligned}
S_{-3,1}(N)= & (-1)^{N} \mathbf{M}\left[\frac{\mathrm{Li}_{3}(x)}{1+x}\right](N+1)+\zeta_{2} S_{-2}(N)-\zeta_{3} S_{-1}(N)-\frac{3}{5} \zeta_{2}^{2}+2 \mathrm{Li}_{4}\left(\frac{1}{2}\right) \\
& +\frac{3}{4} \zeta_{3} \ln (2)-\frac{1}{2} \zeta_{2} \ln ^{2}(2)+\frac{1}{12} \ln ^{4}(2) \\
S_{-2,1}(N)= & -(-1)^{N} \mathbf{M}\left[\frac{\mathrm{Li}_{2}(x)}{1+x}\right](N+1)+\zeta_{2} S_{-1}(N)-\frac{5}{8} \zeta_{3}+\zeta_{2} \ln (2) \\
S_{-2,2}(N)= & -(-1)^{N} \mathbf{M}\left[\frac{1}{1+x}\left(2 \mathrm{Li}_{3}(x)-\ln (x)\left(\operatorname{Li}_{2}(x)+\zeta_{2}\right)\right)\right](N+1)+\zeta_{2} S_{-2}(N) \\
& +2 \zeta_{3} S_{-1}(N)+\frac{71}{40} \zeta_{2}^{2}-4 \mathrm{Li}_{4}\left(\frac{1}{2}\right)-\frac{3}{2} \zeta_{3} \ln (2)+\zeta_{2} \ln ^{2}(2)-\frac{1}{6} \ln ^{4}(2) \\
S_{2,1}(N)= & \mathbf{M}\left[\left(\frac{\mathrm{Li}_{2}(x)}{1-x}\right)_{+}\right](N+1)+\zeta_{2} S_{1}(N) \\
S_{3,1}(N)= & -\frac{1}{2} \mathbf{M}\left[\frac{\mathrm{Li}_{2}(x) \ln (x)}{1-x}\right](N+1)+\zeta_{2} S_{2}(N)-\frac{1}{4} S_{2}^{2}(N)-\frac{1}{4} S_{4}(N)-\frac{3}{20} \zeta_{2}^{2}
\end{aligned}
$$

Two triple sums contribute :

$$
\begin{aligned}
S_{-2,1,1}(N)= & -(-1)^{N} \mathbf{M}\left[\frac{S_{1,2}(x)}{1+x}\right](N+1)+\zeta_{3} S_{-1}(N)-\operatorname{Li}_{4}\left(\frac{1}{2}\right)+\frac{1}{8} \zeta_{2}^{2}+\frac{1}{8} \zeta_{3} \ln (2) \\
& +\frac{1}{4} \zeta_{2} \ln ^{2}(2)-\frac{1}{24} \ln ^{4}(2) \\
S_{2,1,1}(N)= & \mathbf{M}\left[\left(\frac{S_{1,2}(x)}{1-x}\right)_{+}\right](N+1)+\zeta_{3} S_{1}(N) .
\end{aligned}
$$

For the analytic continuation it is sufficient to determine $\mathbf{M}[f(x)](N)$ for complex values of $N$ since

$$
\frac{\partial^{k}}{\partial N^{k}} \mathbf{M}[f(x)](N)=\mathbf{M}\left[\ln ^{k}(x) f(x)\right](N)
$$

is easily obtained analytically. Therefore we will not count the associated derivatives as genuinely new functions. Besides Euler's $\psi$-function the Mellin transforms of five further basic functions,

$$
\frac{\operatorname{Li}_{2}(x)}{1-x}, \quad \frac{\operatorname{Li}_{2}(x)}{1+x}, \quad \frac{S_{1,2}(x)}{1-x}, \quad \frac{S_{1,2}(x)}{1+x}, \quad \frac{\operatorname{Li}_{3}(x)}{1+x}
$$

are sufficient to express the different Wilson coefficients dealt with in the present paper. The Mellin transforms of these functions were calculated in [12] and are denoted by $A_{k}(N)$ with 
$k=18,3,21,8,18,6$. In the appendices we furthermore refer to the functions $A_{5}(N)$ and $A_{22}(N)$, which are given by

$$
\begin{aligned}
A_{5}(N) & =\frac{\partial}{\partial N} A_{3}(N), \\
A_{22}(N) & =\frac{\partial}{\partial N} A_{18}(N) .
\end{aligned}
$$

The analytic continuation to $N \epsilon \mathrm{C}$ is unique [28]. The hadronic cross sections are obtained calculating the inverse Mellin transform of $(23,33)$ by a numerical contour integral

$$
F(x)=\frac{1}{2 \pi i} \int_{c-i \infty}^{c+i \infty} d N x^{-N} \mathbf{M}[F(x)](N) .
$$

Here the parameter $c$ is the intersection of the contour and the real axis and is chosen right to the rightmost singularity of the function $\mathbf{M}[F(x)](N)$. The shape of the contour can be deformed at our convenience covering all singularities of $\mathbf{M}[F(x)](N)$.

\section{Conclusion}

We have systematically analyzed the mathematical structure behind the NNLO coefficient functions for the unpolarized and polarized Drell-Yan process and hadronic scalar and pseudo-scalar Higgs boson production using Mellin moment techniques. Use of various algebraic and structural identities, which relate the finite harmonic sums, reduces the complexity of the results from around 80 functions to only five basic functions, the $\psi$-function and a few derivatives thereof. This is very useful both for the understanding of the nature of higher order corrections in the massless case and yields expressions which allow to perform fast numerical calculations at high precision for phenomenological applications and fits to data. The same structures are found in the case of polarized and unpolarized 2-loop fragmentation functions [21]. Together with the results of [20] for the Wilson coefficients for unpolarized and polarized deeply inelastic scattering it is now shown that these structures are in common for all known massless 2-loop Wilson coefficients.

\section{Acknowledgment.}

We thank S. Moch for discussions. V.R. would like to thank DESY for their kind hospitality extended to him. This paper was supported in part by DFG Sonderforschungsbereich Transregio 9, Computergestützte Theoretische Physik, and EU grant HPRN-CT-2000-00149. 


\section{A.1 Unpolarized Drell-Yan Coefficient Functions}

In this appendix we present the Mellin transforms of the coefficient functions for the unpolarized Drell-Yan Process. We used the same notation as in Refs. [5] and refer either to single harmonic sums $S_{k}(N)$ or special functions, $A_{l}(N)$. To avoid the occurrence of boundary terms in case of $S_{-k}(N)$ we use the functions $\beta^{(k-1)}(N), \quad k \geq 1$, instead, with $N \in \mathbf{C}$, outside the respective single poles of the functions below.

$$
\begin{aligned}
& \Delta_{a b}=\Delta_{a b}^{(0)}+a_{s} \Delta_{a b}^{(1)}+a_{s}^{2} \Delta_{a b}^{(2)} \\
& \Delta_{q \bar{q}}^{(0)}=1 \\
& \Delta_{q \bar{q}}^{(1)}=C_{F}\left[\left(-\frac{4}{(N+1)}-\frac{4}{N}-8 S_{1}(N-1)+6\right) \ln \left(\frac{Q^{2}}{\mu^{2}}\right)-\frac{4}{N^{2}}+8 \frac{S_{1}(N)}{N}\right. \\
& \left.-\frac{4}{(N+1)^{2}}+16 \zeta_{2}-16+8 \frac{S_{1}(N+1)}{(N+1)}+8 S_{1}^{2}(N-1)\right] \\
& \Delta_{q g}^{(1)}=T_{f}\left[\left(-\frac{4}{(N+1)}+\frac{2}{N}+\frac{4}{(N+2)}\right) \ln \left(\frac{Q^{2}}{\mu^{2}}\right)+\frac{2}{N^{2}}+\frac{6}{(N+1)}+\frac{1}{N}\right. \\
& \left.-\frac{4}{(N+1)^{2}}-\frac{7}{(N+2)}+8 \frac{S_{1}(N+1)}{(N+1)}+\frac{4}{(N+2)^{2}}-4 \frac{S_{1}(N)}{N}-8 \frac{S_{1}(N+2)}{(N+2)}\right] \\
& \Delta_{q \bar{q}}^{(2), S+V}=C_{F}^{2}\left[\left(18+32 S_{1}^{2}(N-1)+32 S_{2}(N-1)-32 \zeta_{2}-48 S_{1}(N-1)\right)\right. \\
& \times \ln ^{2}\left(\frac{Q^{2}}{\mu^{2}}\right)+\left(\left(128-192 S_{2}(N-1)+64 \zeta_{2}\right) S_{1}(N-1)+48 S_{1}^{2}(N-1)+176 \zeta_{3}\right. \\
& \left.+48 S_{2}(N-1)-93+24 \zeta_{2}-64 S_{1}^{3}(N-1)-128 S_{3}(N-1)\right) \ln \left(\frac{Q^{2}}{\mu^{2}}\right)+\left(-256 \zeta_{3}\right. \\
& \left.+256 S_{3}(N-1)\right) S_{1}(N-1)+\left(-64 \zeta_{2}-128+192 S_{2}(N-1)\right) S_{1}^{2}(N-1) \\
& +32 S_{1}^{4}(N-1)+\left(-64 \zeta_{2}-128\right) S_{2}(N-1)-70 \zeta_{2}-60 \zeta_{3}+\frac{511}{4}+192 S_{4}(N-1) \\
& \left.+\frac{8}{5} \zeta_{2}^{2}+96 S_{2}^{2}(N-1)\right] \\
& +C_{A} C_{F}\left[\left(\frac{44}{3} S_{1}(N-1)-11\right) \ln ^{2}\left(\frac{Q^{2}}{\mu^{2}}\right)+\left(\left(16 \zeta_{2}-\frac{536}{9}\right) S_{1}(N-1)\right.\right. \\
& \left.-24 \zeta_{3}+\frac{193}{3}-\frac{88}{3} S_{1}^{2}(N-1)-\frac{88}{3} S_{2}(N-1)\right) \ln \left(\frac{Q^{2}}{\mu^{2}}\right)+\left(-56 \zeta_{3}-\frac{176}{3} \zeta_{2}\right.
\end{aligned}
$$




$$
\begin{aligned}
& \left.+\frac{176}{3} S_{2}(N-1)+\frac{1616}{27}\right) S_{1}(N-1)+\left(-16 \zeta_{2}+\frac{536}{9}\right) S_{1}^{2}(N-1)+\frac{176}{9} S_{1}^{3}(N-1) \\
& \left.+\left(-16 \zeta_{2}+\frac{536}{9}\right) S_{2}(N-1)-\frac{12}{5} \zeta_{2}^{2}+28 \zeta_{3}+\frac{352}{9} S_{3}(N-1)-\frac{1535}{12}+\frac{592}{9} \zeta_{2}\right] \\
& +C_{F} n_{f}\left[\left(2-\frac{8}{3} S_{1}(N-1)\right) \ln ^{2}\left(\frac{Q^{2}}{\mu^{2}}\right)+\left(\frac{16}{3} S_{2}(N-1)-\frac{34}{3}+\frac{16}{3} S_{1}^{2}(N-1)\right.\right. \\
& \left.+\frac{80}{9} S_{1}(N-1)\right) \ln \left(\frac{Q^{2}}{\mu^{2}}\right)+\left(\frac{32}{3} \zeta_{2}-\frac{224}{27}-\frac{32}{3} S_{2}(N-1)\right) S_{1}(N-1)+8 \zeta_{3} \\
& \left.-\frac{112}{9} \zeta_{2}-\frac{64}{9} S_{3}(N-1)+\frac{127}{6}-\frac{80}{9} S_{1}^{2}(N-1)-\frac{32}{9} S_{1}^{3}(N-1)-\frac{80}{9} S_{2}(N-1)\right] \\
& \Delta_{q \bar{q}}^{(2), C_{A}}=C_{A} C_{F}\left[\frac{208}{3} \zeta_{2}+\frac{98}{3} \zeta_{3}+\left(40 A_{18}(N-1)+\frac{272}{3} \zeta_{2}-16 \zeta_{3}-\frac{272}{3} S_{2}(N-1)\right.\right. \\
& \left.+16 S_{3}(N-1)\right) S_{1}(N-1)-\frac{52}{3 N^{2}}-\frac{446}{27 N}-\frac{218}{3(N+1)^{2}}+\frac{2278}{27(N+1)} \\
& -\frac{98}{3} S_{3}(N-1)-12 S_{4}(N-1)+\frac{110}{3 N^{3}}+\frac{110}{3(N+1)^{3}}+\frac{8}{3} A_{18}(N-1) \\
& -24 A_{21}(N-1)+\left(\frac{22}{3(N+1)}+\frac{22}{3 N}\right) \ln ^{2}\left(\frac{Q^{2}}{\mu^{2}}\right)+\frac{16 \zeta_{2}}{N^{2}}+\frac{68 \zeta_{2}}{3 N}-\frac{24 \zeta_{3}}{N} \\
& +\frac{16 \zeta_{2}}{(N+1)^{2}}+\frac{20 \zeta_{2}}{3(N+1)}-24 \frac{\zeta_{3}}{(N+1)}-4 \frac{S_{3}(N+1)}{(N+1)}-4 \frac{S_{3}(N)}{N}+\frac{88 S_{1}^{2}(N+1)}{3(N+1)} \\
& +\frac{88 S_{1}^{2}(N)}{3 N}+\left(-48 \frac{\zeta_{2}}{N}+32 \frac{S_{2}(N)}{N}-\frac{176}{3 N^{2}}+\frac{152}{9 N}\right) S_{1}(N)+\left(32 \frac{S_{2}(N+1)}{(N+1)}\right. \\
& \left.-48 \frac{\zeta_{2}}{(N+1)}+\frac{956}{9(N+1)}-\frac{176}{3(N+1)^{2}}\right) S_{1}(N+1)+16 \frac{A_{18}(N)}{N}+16 \frac{A_{18}(N+1)}{(N+1)} \\
& +\left(-28 \zeta_{2}+28 S_{2}(N-1)\right) S_{1}^{2}(N-1)+\left(-\frac{208}{3}+4 \zeta_{2}\right) S_{2}(N-1)+\left(-\frac{8}{(N+1)^{2}}\right. \\
& \left.-\frac{32}{3(N+1)}\right) S_{2}(N+1)+\left(-\frac{8}{N^{2}}-\frac{56}{3 N}\right) S_{2}(N)+\frac{4}{5} \zeta_{2}^{2}+\left(\left(16 \zeta_{2}\right.\right. \\
& \left.-16 S_{2}(N-1)\right) S_{1}(-1+N)+16 \zeta_{3}-\frac{140}{3} \zeta_{2}-8 \frac{S_{2}(N+1)}{(N+1)}-\frac{88 S_{1}(N+1)}{3(N+1)}+16 \frac{\zeta_{2}}{N} \\
& -\frac{496}{9(N+1)}+\frac{88}{3 N^{2}}+\frac{140}{3} S_{2}(N-1)+\frac{88}{3(N+1)^{2}}-\frac{76}{9 N}-16 A_{18}(N-1)
\end{aligned}
$$




$$
\begin{aligned}
& \left.\left.-\frac{88 S_{1}(N)}{3 N}+16 \frac{\zeta_{2}}{(N+1)}-8 \frac{S_{2}(N)}{N}-16 S_{3}(N-1)\right) \ln \left(\frac{Q^{2}}{\mu^{2}}\right)\right] \\
& \Delta_{q \bar{q}}^{(2), C_{F}}=C_{F}^{2}\left[-112 \zeta_{2}+\left(-\frac{24}{N^{2}}+32 \zeta_{2}-\frac{40}{N}+32 \frac{S_{1}(N+1)}{(N+1)}-\frac{8}{(N+1)}\right.\right. \\
& \left.+32 \frac{S_{1}(N)}{N}-32 S_{2}(N-1)-\frac{24}{(N+1)^{2}}\right) \ln ^{2}\left(\frac{Q^{2}}{\mu^{2}}\right)+\frac{104}{N^{2}}-\frac{72}{N} \\
& -\frac{48}{(N+1)^{2}}+\frac{48}{(N+1)}-216 S_{4}(N-1)-104 S_{2}^{2}(N-1)-\frac{16}{N^{3}}+\frac{48}{(N+1)^{3}} \\
& -16 A_{21}(N-1)-\frac{100}{N^{4}}-\frac{100}{(N+1)^{4}}+\left(232 \frac{\zeta_{2}}{(N+1)}-\frac{112}{(N+1)^{2}}\right. \\
& \left.+\frac{192}{(N+1)^{3}}-104 \frac{S_{2}(N+1)}{(N+1)}-\frac{12}{(N+1)}\right) S_{1}(N+1)-120 \frac{\zeta_{2}}{N^{2}}-24 \frac{\zeta_{2}}{N}-24 \frac{\zeta_{3}}{N} \\
& -120 \frac{\zeta_{2}}{(N+1)^{2}}+16 \frac{\zeta_{2}}{(N+1)}-24 \frac{\zeta_{3}}{(N+1)}+24 \frac{S_{3}(N+1)}{(N+1)}+24 \frac{S_{3}(N)}{N}-8 \frac{A_{18}(N)}{N} \\
& -8 \frac{A_{18}(N+1)}{(N+1)}+\left(\frac{64}{N^{2}}+\frac{192}{N^{3}}-104 \frac{S_{2}(N)}{N}+232 \frac{\zeta_{2}}{N}-\frac{256}{N}\right) S_{1}(N)+\left(288 \zeta_{2}\right. \\
& \left.-288 S_{2}(N-1)\right) S_{1}^{2}(N-1)+64 \frac{S_{1}^{3}(N)}{N}+64 \frac{S_{1}^{3}(N+1)}{(N+1)}+\left(-\frac{156}{(N+1)^{2}}\right. \\
& \left.+\frac{64}{(N+1)}\right) S_{1}^{2}(N+1)+\frac{552}{5} \zeta_{2}^{2}+\left(-\frac{64}{N}-\frac{156}{N^{2}}\right) S_{1}^{2}(N)+\left(\left(-256 \zeta_{2}\right.\right. \\
& \left.+256 S_{2}(N-1)\right) S_{1}(N-1)+\left(\frac{144}{(N+1)^{2}}-\frac{16}{(N+1)}\right) S_{1}(N+1)+\left(\frac{144}{N^{2}}\right. \\
& \left.+\frac{112}{N}\right) S_{1}(N)+32 \frac{S_{2}(N)}{N}+\frac{24}{(N+1)^{2}}-48 S_{2}(N-1)+32 \frac{S_{2}(N+1)}{(N+1)}-\frac{56}{N^{2}} \\
& +32 A_{18}(N-1)-\frac{72}{N^{3}}+48 \zeta_{2}+160 S_{3}(N-1)-96 \frac{\zeta_{2}}{N}-\frac{72}{(N+1)^{3}}-96 \frac{S_{1}^{2}(N)}{N} \\
& \left.-160 \zeta_{3}-96 \frac{S_{1}^{2}(N+1)}{(N+1)}+\frac{16}{(N+1)}+\frac{120}{N}-96 \frac{\zeta_{2}}{(N+1)}\right) \ln \left(\frac{Q^{2}}{\mu^{2}}\right)+(112+ \\
& \left.80 \zeta_{2}\right) S_{2}(N-1)+\left(-\frac{16}{(N+1)}+\frac{60}{(N+1)^{2}}\right) S_{2}(N+1)+\left(\frac{24}{N}+\frac{60}{N^{2}}\right) S_{2}(N) \\
& \left.+\left(256 \zeta_{3}-256 S_{3}(N-1)-32 A_{18}(N-1)\right) S_{1}(N-1)\right]
\end{aligned}
$$




$$
\begin{aligned}
& \Delta_{q \bar{q}, A \bar{A}}^{(2)}=C_{F} n_{f}\left[\left(-\frac{4}{3 N}-\frac{4}{3(N+1)}\right) \ln ^{2}\left(\frac{Q^{2}}{\mu^{2}}\right)+\left(-\frac{16}{3 N^{2}}-\frac{32}{3} S_{2}(N-1)\right.\right. \\
& \left.-\frac{8}{9 N}+\frac{88}{9(N+1)}+\frac{16}{3} \frac{S_{1}(N)}{N}-\frac{16}{3(N+1)^{2}}+\frac{16}{3} \frac{S_{1}(N+1)}{(N+1)}+\frac{32}{3} \zeta_{2}\right) \ln \left(\frac{Q^{2}}{\mu^{2}}\right) \\
& +\left(\frac{56}{3} S_{2}(N-1)-\frac{56}{3} \zeta_{2}\right) S_{1}(N-1)+\left(-\frac{176}{9(N+1)}+\frac{32}{3(N+1)^{2}}\right) S_{1}(N+1) \\
& +\left(\frac{16}{9 N}+\frac{32}{3 N^{2}}\right) S_{1}(N)-\frac{40}{3} \zeta_{2}-\frac{8}{3} \zeta_{3}+\frac{4}{3 N^{2}}+\frac{188}{27 N}-\frac{20}{3 N^{3}}+\frac{40}{3} S_{2}(N-1) \\
& -\frac{8 \zeta_{2}}{3 N}-\frac{16 S_{1}^{2}(N)}{3 N}+\frac{44}{3(N+1)^{2}}-\frac{8}{3} A_{18}(N-1)+\frac{8}{3} S_{3}(N-1)-\frac{20}{3(N+1)^{3}} \\
& \left.-\frac{16 S_{1}^{2}(N+1)}{3(N+1)}-\frac{8 \zeta_{2}}{3(N+1)}-\frac{412}{27(N+1)}+\frac{8 S_{2}(N+1)}{3(N+1)}+\frac{8 S_{2}(N)}{3 N}\right] \\
& \Delta_{q \bar{q}, A \bar{A}}^{(2)}=\Delta_{q \bar{q}, A \bar{D}}^{(2)} \\
& =C_{F}\left(C_{F}-\frac{C_{A}}{2}\right)\left[-24 \zeta_{2}+18 \zeta_{3}-\frac{32}{N^{2}}+\frac{94}{N}+\frac{30}{(N+1)^{2}}-\frac{78}{(N+1)}\right. \\
& -18 S_{3}(N-1)-2 S_{4}(N-1)+6 S_{2}^{2}(N-1)+\frac{8}{N^{3}}+\frac{8}{(N+1)^{3}}-12 A_{18}(N-1) \\
& +32 A_{21}(N-1)+\frac{12}{N^{4}}+\frac{12}{(N+1)^{4}}+12 A_{22}(N-1)+\left(-\frac{16}{N^{3}}-\frac{16}{N^{2}}\right. \\
& \left.+8 \frac{S_{2}(N)}{N}+\frac{64}{N}-8 \frac{\zeta_{2}}{N}\right) S_{1}(N)+\left(-16 \zeta_{2}+16 S_{2}(N-1)\right) S_{1}^{2}(N-1) \\
& +\left(24+16 \zeta_{2}\right) S_{2}(N-1)-4 \frac{\zeta_{2}}{N}+12 \frac{\zeta_{3}}{N}-4 \frac{\zeta_{2}}{(N+1)}+12 \frac{\zeta_{3}}{(N+1)}+4 \frac{S_{2}(N+1)}{(N+1)} \\
& +4 \frac{S_{2}(N)}{N}-12 \frac{S_{3}(N+1)}{(N+1)}-12 \frac{S_{3}(N)}{N}-8 \frac{A_{18}(N+1)}{(N+1)}-8 \frac{A_{18}(N)}{N}+\left(\left(-16 S_{2}(N-1)\right.\right. \\
& \left.+16 \zeta_{2}\right) S_{1}(N-1)-16 A_{18}(N-1)+12 \zeta_{2}+\frac{28}{(N+1)}+\frac{8}{(N+1)^{3}}+8 \frac{\zeta_{2}}{N} \\
& -8 \frac{S_{2}(N+1)}{(N+1)}-8 \frac{S_{2}(N)}{N}+\frac{8}{(N+1)^{2}}+\frac{8}{N^{3}}+\frac{8}{N^{2}}-12 S_{2}(N-1)-\frac{32}{N} \\
& \left.+8 \frac{\zeta_{2}}{(N+1)}\right) \ln \left(\frac{Q^{2}}{\mu^{2}}\right)-\frac{106}{5} \zeta_{2}^{2}+\left(24 \zeta_{3}+12 S_{2}(N-1)-24 S_{3}(N-1)\right.
\end{aligned}
$$




$$
\begin{aligned}
& \left.-12 \zeta_{2}\right) S_{1}(N-1)+\left(-\frac{16}{(N+1)^{3}}-\frac{56}{(N+1)}+8 \frac{S_{2}(N+1)}{(N+1)}-8 \frac{\zeta_{2}}{(N+1)}\right. \\
& \left.\left.-\frac{16}{(N+1)^{2}}\right) S_{1}(N+1)\right] \\
& \Delta_{q \bar{q}, B \bar{B}}^{(2)}=C_{F}\left[\frac{16}{3 N^{3}}-\frac{8}{N^{2}}+\frac{40}{3 N}+\frac{32}{3(N+1)^{3}}-\frac{32}{3(N+1)^{2}}+\frac{16}{3(N+2)^{3}}\right. \\
& \left.-\frac{8}{(N+2)^{2}}-\frac{40}{3(N+2)}+\frac{32 \beta^{(1)}(N+1)}{3 N}+\frac{64 \beta^{(1)}(N+2)}{3(N+1)}+\frac{32 \beta^{(1)}(3+N)}{3(N+2)}\right] \\
& \Delta_{q \bar{q}, B \bar{C}}^{(2)}=\Delta_{q \bar{q}, B \bar{D}}^{(2)} \\
& =C_{F}\left(C_{F}-\frac{C_{A}}{2}\right)\left[-\frac{36}{N^{2}}+\frac{54}{N}-\frac{44}{(N+1)^{2}}-\frac{28}{(N+1)}-\frac{26}{(N+2)}+\frac{24}{N^{3}}\right. \\
& -\frac{32}{(N+1)^{3}}+\left(\frac{16}{(N+2)^{2}}+\frac{36}{(N+2)}\right) S_{2}(N+2)-\frac{8}{N^{4}}-\frac{32}{(N+1)^{4}} \\
& -\frac{60}{(N+2)^{3}}-48 \frac{\beta(N+1) \zeta_{2}}{N}-\frac{8}{(N+2)^{4}}-96 \frac{S_{1}(N+1) \beta^{(1)}(N+2)}{(N+1)}-16 \frac{\zeta_{2}}{N^{2}} \\
& +36 \frac{\zeta_{2}}{N}-48 \frac{\zeta_{2}}{(N+1)^{2}}-16 \frac{\zeta_{2}}{(N+2)^{2}}-36 \frac{\zeta_{2}}{(N+2)}+48 \frac{S_{2}(N+1)}{(N+1)^{2}}+16 \frac{\beta^{(1)}(N+1)}{N^{2}} \\
& -24 \frac{\beta^{(1)}(N+1)}{N}+32 \frac{\beta^{(1)}(N+2)}{(N+1)^{2}}-48 \frac{\beta^{(1)}(N+2)}{(N+1)}+16 \frac{\beta^{(1)}(3+N)}{(N+2)^{2}}+4 \frac{\beta^{(2)}(N+1)}{N} \\
& +8 \frac{\beta^{(2)}(N+2)}{(N+1)}+4 \frac{\beta^{(2)}(3+N)}{(N+2)}+96 \frac{A_{3}(N+1)}{(N+1)}+48 \frac{A_{3}(N+2)}{(N+2)}+48 \frac{A_{3}(N)}{N} \\
& -48 \frac{S_{1}(N+2) \beta^{(1)}(3+N)}{(N+2)}-48 \frac{S_{1}(N) \beta^{(1)}(N+1)}{N}-96 \frac{\beta(N+2) \zeta_{2}}{(N+1)}-48 \frac{\beta(3+N) \zeta_{2}}{(N+2)} \\
& \left.-24 \frac{\beta^{(1)}(3+N)}{(N+2)}+\left(\frac{16}{N^{2}}-\frac{36}{N}\right) S_{2}(N)\right] \\
& \Delta_{q g}^{(2), C_{A}}=C_{A} T_{f}\left[-\frac{236}{3 N^{2}}+\frac{539}{9 N}+\frac{32}{3(N+1)^{2}}-\frac{1226}{9(N+1)}+\frac{914}{9(N+2)^{2}}\right. \\
& +\frac{1837}{27(N+2)}+\left(\frac{20}{N^{2}}-\frac{16}{N}\right) S_{2}(N)-\frac{10}{N^{3}}-\frac{8}{(N+1)^{3}}-\frac{36}{N^{4}}-\frac{80}{(N+1)^{4}} \\
& +24 \frac{A_{18}(N+2)}{(N+2)}+\frac{32 \zeta_{2}}{3(N-1)}-\frac{692}{3(N+2)^{3}}-\frac{52 S_{1}^{3}(N+2)}{3(N+2)}+\frac{32 S_{1}^{2}(N-1)}{3(N-1)}
\end{aligned}
$$




$$
\begin{aligned}
& -\frac{176 S_{1}(N-1)}{9(N-1)}-\frac{32 S_{2}(N-1)}{3(N-1)}+\left(\frac{296}{3} \frac{1}{(N+2)}-\frac{8}{(N+2)^{2}}\right) S_{2}(N+2) \\
& -20 \frac{\zeta_{2}}{N^{2}}+40 \frac{\zeta_{2}}{N}-10 \frac{\zeta_{3}}{N}-80 \frac{\zeta_{2}}{(N+1)^{2}}+28 \frac{\zeta_{2}}{(N+1)}+20 \frac{\zeta_{3}}{(N+1)}+8 \frac{\zeta_{2}}{(N+2)^{2}} \\
& -\frac{224 \zeta_{2}}{3(N+2)}-28 \frac{\zeta_{3}}{(N+2)}+32 \frac{S_{3}(N+1)}{3(N+1)}-\frac{8 S_{3}(N+2)}{3(N+2)}-\frac{16 S_{3}(N)}{3 N} \\
& +8 \frac{\beta^{(1)}(N+1)}{N^{2}}-8 \frac{\beta^{(1)}(N+1)}{N}+16 \frac{\beta^{(1)}(N+2)}{(N+1)^{2}}-40 \frac{\beta^{(1)}(N+2)}{(N+1)}+16 \frac{\beta^{(1)}(3+N)}{(N+2)^{2}} \\
& -4 \frac{\beta^{(2)}(N+1)}{N}-8 \frac{\beta^{(2)}(N+2)}{(N+1)}-8 \frac{\beta^{(2)}(3+N)}{(N+2)}-24 \frac{A_{18}(N+1)}{(N+1)}+12 \frac{A_{18}(N)}{N} \\
& -32 \frac{\beta^{(1)}(3+N)}{(N+2)}+\left(-\frac{12}{N^{2}}-\frac{58}{3 N}+\frac{8}{(N+1)^{2}}-\frac{8}{3(N+1)}-\frac{112}{(N+2)^{2}}\right. \\
& +\frac{146}{9(N+2)}-\frac{16}{N^{3}}-\frac{48}{(N+1)^{3}}+\left(\frac{284}{3(N+2)}-\frac{16}{(N+2)^{2}}\right) S_{1}(N+2) \\
& -\frac{32 S_{1}(N-1)}{3(N-1)}+4 \frac{\zeta_{2}}{N}-8 \frac{\zeta_{2}}{(N+1)}+8 \frac{\zeta_{2}}{(N+2)}+8 \frac{S_{2}(N+1)}{(N+1)}-8 \frac{S_{2}(N+2)}{(N+2)} \\
& -4 \frac{S_{2}(N)}{N}+8 \frac{\beta^{(1)}(N+1)}{N}+16 \frac{\beta^{(1)}(N+2)}{(N+1)}-24 \frac{S_{1}^{2}(N+1)}{(N+1)}+24 \frac{S_{1}^{2}(N+2)}{(N+2)} \\
& +12 \frac{S_{1}^{2}(N)}{N}+\left(\frac{80}{(N+1)^{2}}-\frac{72}{(N+1)}\right) S_{1}(N+1)+\left(-\frac{12}{N}+\frac{8}{N^{2}}\right) S_{1}(N) \\
& \left.+16 \frac{\beta^{(1)}(3+N)}{(N+2)}+\frac{88}{9(N-1)}\right) \ln \left(\frac{Q^{2}}{\mu^{2}}\right)+\left(72 \frac{\zeta_{2}}{(N+1)}-\frac{32}{(N+1)^{2}}-32 \frac{\beta^{(1)}(N+2)}{(N+1)}\right. \\
& \left.-68 \frac{S_{2}(N+1)}{(N+1)}-\frac{50}{3(N+1)}+\frac{112}{(N+1)^{3}}\right) S_{1}(N+1)+\left(-\frac{76}{(N+1)}\right. \\
& \left.+\frac{80}{(N+1)^{2}}\right) S_{2}(N+1)+\left(-8 \frac{S_{1}(N+2)}{(N+2)}+\frac{16}{(N+1)}-4 \frac{S_{1}(N)}{N}-\frac{62}{3(N+2)}\right. \\
& \left.-\frac{16}{(N+1)^{2}}-\frac{4}{N^{2}}+8 \frac{S_{1}(N+1)}{(N+1)}+\frac{8}{3(N-1)}+\frac{2}{N}\right) \ln ^{2}\left(\frac{Q^{2}}{\mu^{2}}\right)+\left(-36 \frac{\zeta_{2}}{N}\right. \\
& \left.+\frac{140}{3 N}+34 \frac{S_{2}(N)}{N}-16 \frac{\beta^{(1)}(N+1)}{N}+\frac{20}{N^{2}}+\frac{24}{N^{3}}\right) S_{1}(N)-\frac{26 S_{1}^{3}(N)}{3 N}
\end{aligned}
$$




$$
\begin{aligned}
& +\frac{52 S_{1}^{3}(N+1)}{3(N+1)}+\frac{116}{27(N-1)}+\left(60 \frac{S_{2}(N+2)}{(N+2)}-32 \frac{\beta^{(1)}(3+N)}{(N+2)}-\frac{148}{9(N+2)}\right. \\
& \left.+\frac{260}{(N+2)^{2}}-\frac{16}{(N+2)^{3}}-64 \frac{\zeta_{2}}{(N+2)}\right) S_{1}(N+2)+\left(\frac{84}{(N+1)}\right. \\
& \left.-\frac{88}{(N+1)^{2}}\right) S_{1}^{2}(N+1)+\left(-\frac{4}{N^{2}}+\frac{8}{N}\right) S_{1}^{2}(N)+\left(\frac{24}{(N+2)^{2}}\right. \\
& \left.\left.-\frac{308}{3(N+2)}\right) S_{1}^{2}(N+2)\right] \\
& \Delta_{q g}^{(2), C_{F}}=C_{F} T_{f}\left[\frac{31}{N^{2}}-\frac{157}{2 N}-\frac{201}{(N+1)^{2}}+\frac{221}{(N+1)}+\frac{174}{(N+2)^{2}}\right. \\
& -\frac{305}{2(N+2)}-\frac{11}{N^{3}}+\frac{60}{(N+1)^{3}}+\frac{34}{N^{4}}-\frac{68}{(N+1)^{4}}-32 \frac{A_{18}(N+2)}{(N+2)} \\
& -\frac{4}{(N+2)^{3}}+\left(24 \frac{S_{1}(N+1)}{(N+1)}+\frac{24}{(N+2)^{2}}-\frac{12}{(N+1)^{2}}+\frac{6}{N^{2}}+\frac{12}{(N+1)}\right. \\
& \left.-12 \frac{S_{1}(N)}{N}-\frac{3}{N}-24 \frac{S_{1}(N+2)}{(N+2)}\right) \ln ^{2}\left(\frac{Q^{2}}{\mu^{2}}\right)+\frac{104}{(N+2)^{4}}-\frac{140 S_{1}^{3}(N+2)}{3(N+2)} \\
& +28 \frac{\zeta_{2}}{N^{2}}-34 \frac{\zeta_{2}}{N}+16 \frac{\zeta_{3}}{N}-56 \frac{\zeta_{2}}{(N+1)^{2}}+168 \frac{\zeta_{2}}{(N+1)}-32 \frac{\zeta_{3}}{(N+1)}+80 \frac{\zeta_{2}}{(N+2)^{2}} \\
& -136 \frac{\zeta_{2}}{(N+2)}+48 \frac{\zeta_{3}}{(N+2)}-2 \frac{S_{2}(N)}{N^{2}}-\frac{80 S_{3}(N+1)}{3(N+1)}+\frac{32 S_{3}(N+2)}{3(N+2)}+\frac{40 S_{3}(N)}{3 N} \\
& +16 \frac{\beta^{(1)}(N+1)}{N^{2}}-16 \frac{\beta^{(1)}(N+1)}{N}-32 \frac{\beta^{(1)}(N+2)}{(N+1)^{2}}+32 \frac{\beta^{(1)}(N+2)}{(N+1)}+32 \frac{\beta^{(1)}(3+N)}{(N+2)^{2}} \\
& +32 \frac{A_{18}(N+1)}{(N+1)}+\left(\left(\frac{80}{(N+1)^{2}}-\frac{136}{(N+1)}\right) S_{1}(N+1)+\left(\frac{32}{N}-\frac{40}{N^{2}}\right) S_{1}(N)\right. \\
& +\left(-\frac{128}{(N+2)^{2}}+\frac{92}{(N+2)}\right) S_{1}(N+2)+\frac{22}{(N+2)}-\frac{92}{(N+2)^{2}}+\frac{64}{(N+2)^{3}} \\
& +72 \frac{S_{1}^{2}(N+2)}{(N+2)}+\frac{16}{N^{3}}-\frac{68}{(N+1)}+\frac{24}{N}-4 \frac{S_{2}(N)}{N}+80 \frac{1}{(N+1)^{2}}-\frac{32}{(N+1)^{3}} \\
& +36 \frac{S_{1}^{2}(N)}{N}-\frac{10}{N^{2}}+32 \frac{\zeta_{2}}{N}+64 \frac{\zeta_{2}}{(N+2)}-64 \frac{\zeta_{2}}{(N+1)}+8 \frac{S_{2}(N+1)}{(N+1)}-72 \frac{S_{1}^{2}(N+1)}{(N+1)} \\
& \left.-8 \frac{S_{2}(N+2)}{(N+2)}\right) \ln \left(\frac{Q^{2}}{\mu^{2}}\right)-16 \frac{A_{18}(N)}{N}+48 \frac{\beta^{(1)}(3+N)}{(N+2)}+\left(\frac{96}{(N+1)^{3}}+80 \frac{\zeta_{2}}{(N+1)}\right.
\end{aligned}
$$




$$
\begin{aligned}
& \left.+\frac{270}{(N+1)}+28 \frac{S_{2}(N+1)}{(N+1)}-\frac{192}{(N+1)^{2}}\right) S_{1}(N+1)+\left(\frac{16}{(N+1)}\right. \\
& \left.+\frac{4}{(N+1)^{2}}\right) S_{2}(N+1)+\left(\frac{28}{N^{2}}-\frac{48}{N^{3}}-\frac{52}{N}-40 \frac{\zeta_{2}}{N}-14 \frac{S_{2}(N)}{N}\right) S_{1}(N) \\
& +\left(-\frac{176}{(N+2)}-\frac{160}{(N+2)^{3}}+\frac{192}{(N+2)^{2}}-12 \frac{S_{2}(N+2)}{(N+2)}\right. \\
& \left.-96 \frac{\zeta_{2}}{(N+2)}\right) S_{1}(N+2)-\frac{70 S_{1}^{3}(N)}{3 N}+\frac{140 S_{1}^{3}(N+1)}{3(N+1)}+\left(-\frac{46}{N}+\frac{42}{N^{2}}\right) S_{1}^{2}(N) \\
& +\left(\frac{160}{(N+1)}-\frac{84}{(N+1)^{2}}\right) S_{1}^{2}(N+1)+\left(-\frac{126}{(N+2)}+\frac{132}{(N+2)^{2}}\right) S_{1}^{2}(N+2) \\
& \left.+\left(-\frac{14}{(N+2)}-\frac{28}{(N+2)^{2}}\right) S_{2}(N+2)\right] \\
& \Delta_{q \bar{q}, C \bar{C}}^{(2)}=\Delta_{q \bar{q}, D \bar{D}}^{(2)}=\Delta_{q q, C \bar{C}}^{(2)}=\Delta_{q q, D \bar{D}}^{(2)}=\Delta_{\bar{q} \bar{q}, C \bar{C}}^{(2)}=\Delta_{\bar{q} \bar{q}, D \bar{D}}^{(2)} \\
& =C_{F} T_{f}\left[-\frac{230}{3 N^{2}}+\frac{593}{9 N}+\frac{32}{3(N+1)^{2}}-\frac{866}{9(N+1)}-\frac{40}{9(N+2)^{2}}+\frac{703}{27(N+2)}\right. \\
& -\frac{10}{N^{3}}-\frac{50}{(N+1)^{3}}-\frac{36}{N^{4}}-\frac{36}{(N+1)^{4}}+\frac{32 \zeta_{2}}{3(N-1)}-\frac{80}{3(N+2)^{3}} \\
& +\frac{32 S_{1}^{2}(N-1)}{3(N-1)}-\frac{176 S_{1}(N-1)}{9(N-1)}-\frac{32 S_{2}(-1+N)}{3(N-1)}-24 \frac{\zeta_{2}}{N^{2}} \\
& +20 \frac{\zeta_{2}}{N}-24 \frac{\zeta_{2}}{(N+1)^{2}}-20 \frac{\zeta_{2}}{(N+1)}-\frac{32 \zeta_{2}}{3(N+2)}+\frac{32 S_{2}(N+2)}{3(N+2)}-\frac{32 S_{1}^{2}(N+2)}{3(N+2)} \\
& +\left(-\frac{32 S_{1}(N-1)}{3(N-1)}+\left(\frac{16}{(N+1)^{2}}+\frac{8}{(N+1)}\right) S_{1}(N+1)+\left(-\frac{8}{N}+\frac{16}{N^{2}}\right) S_{1}(N)\right. \\
& -\frac{16}{N^{3}}-\frac{16}{(N+2)^{2}}-\frac{16}{(N+1)^{3}}-\frac{88}{9(N+2)}+\frac{88}{9(N-1)}-\frac{24}{(N+1)^{2}} \\
& \left.-\frac{52}{3 N}+\frac{52}{3(N+1)}-\frac{12}{N^{2}}+\frac{32 S_{1}(N+2)}{3(N+2)}\right) \ln \left(\frac{Q^{2}}{\mu^{2}}\right)+\left(-\frac{16}{N^{2}}+\frac{8}{N}\right) S_{1}^{2}(N) \\
& +\left(-\frac{8}{3(N+2)}+\frac{8}{3(N-1)}-\frac{4}{N^{2}}-\frac{4}{(N+1)^{2}}-\frac{2}{(N+1)}+\frac{2}{N}\right) \ln ^{2}\left(\frac{Q^{2}}{\mu^{2}}\right) \\
& +\left(-\frac{104}{3(N+1)}+\frac{32}{(N+1)^{3}}+\frac{48}{(N+1)^{2}}\right) S_{1}(N+1)+\left(\frac{32}{N^{3}}+\frac{104}{3 N}\right.
\end{aligned}
$$




$$
\begin{aligned}
& \left.+\frac{24}{N^{2}}\right) S_{1}(N)+\left(\frac{32}{(N+2)^{2}}+\frac{176}{9(N+2)}\right) S_{1}(N+2)+\left(\frac{20}{(N+1)}\right. \\
& \left.+\frac{24}{(N+1)^{2}}\right) S_{2}(N+1)+\left(24 \frac{1}{N^{2}}-\frac{20}{N}\right) S_{2}(N)+\left(-\frac{16}{(N+1)^{2}}\right. \\
& \left.\left.-\frac{8}{(N+1)}\right) S_{1}^{2}(N+1)+\frac{116}{27(N-1)}\right] \\
& \Delta_{q \bar{q}, C \bar{D}}^{(2)}=-\Delta_{q q, C \bar{D}}^{(2)}=-\Delta_{\bar{q} \bar{q}, C \bar{D}}^{(2)} \\
& =C_{F} T_{f}\left[\frac{80}{N^{2}}-\frac{160}{N}+\frac{64}{(N+1)^{2}}+\frac{160}{(N+1)}-\frac{104}{(N+1)^{3}}+\frac{32}{(N+1)^{4}}\right. \\
& -192 \frac{\beta(N+1) \zeta_{2}}{N}-80 \frac{\zeta_{2}}{N^{2}}+40 \frac{\zeta_{2}}{N}-48 \frac{\zeta_{3}}{N}+8 \frac{\zeta_{2}}{(N+1)^{2}}-32 \frac{\zeta_{2}}{(N+1)} \\
& +24 \frac{\zeta_{3}}{(N+1)}-24 \frac{S_{3}(N+1)}{(N+1)}+48 \frac{S_{3}(N)}{N}+160 \frac{\beta^{(1)}(N+1)}{N^{2}}-80 \frac{\beta^{(1)}(N+1)}{N} \\
& +16 \frac{\beta^{(1)}(N+2)}{(N+1)^{2}}-80 \frac{\beta^{(1)}(N+2)}{(N+1)}+16 \frac{\beta^{(2)}(N+1)}{N}+8 \frac{\beta^{(2)}(N+2)}{(N+1)}+96 \frac{A_{3}(N+1)}{(N+1)} \\
& +192 \frac{A_{3}(N)}{N}-96 \frac{\beta(N+2) \zeta_{2}}{(N+1)}+\left(-24 \frac{\zeta_{2}}{(N+1)}-96 \frac{\beta^{(1)}(N+2)}{(N+1)}\right. \\
& \left.+24 \frac{S_{2}(N+1)}{(N+1)}\right) S_{1}(N+1)+24 \frac{A_{18}(N+1)}{(N+1)}+32 \frac{A_{18}(N-1)}{(N-1)}-192 \frac{\beta(N) \zeta_{2}}{(N-1)} \\
& -48 \frac{A_{18}(N)}{N}+\left(48 \frac{\zeta_{2}}{N}-48 \frac{S_{2}(N)}{N}-192 \frac{\beta^{(1)}(N+1)}{N}\right) S_{1}(N)+192 \frac{A_{3}(N-1)}{(N-1)} \\
& +16 \frac{\beta^{(2)}(N)}{(N-1)}-32 \frac{S_{3}(N-1)}{(N-1)}+32 \frac{\zeta_{3}}{(N-1)}+\left(-\frac{40}{N}+\frac{80}{N^{2}}\right) S_{2}(N) \\
& +\left(-\frac{8}{(N+1)^{2}}+\frac{32}{(N+1)}\right) S_{2}(N+1)+\left(-192 \frac{\beta^{(1)}(N)}{(N-1)}-32 \frac{\zeta_{2}}{(N-1)}\right. \\
& \left.\left.+32 \frac{S_{2}(N-1)}{(N-1)}\right) S_{1}(N-1)\right] \\
& \Delta_{q q, C \bar{E}}^{(2)}=\Delta_{q q, D \bar{F}}^{(2)}=\Delta_{\bar{q} \bar{q}, C \bar{E}}^{(2)}=\Delta_{\bar{q} \bar{q}, D \bar{F}}^{(2)}= \\
& =C_{F}\left(C_{F}-\frac{C_{A}}{2}\right)\left[\frac{18}{N^{2}}-\frac{34}{N}-\frac{14}{(N+1)^{2}}+\frac{34}{(N+1)}-\frac{8}{N^{3}}-\frac{24}{(N+1)^{3}}\right.
\end{aligned}
$$




$$
\begin{aligned}
& -\frac{12}{N^{4}}+\frac{12}{(N+1)^{4}}+\frac{16}{3} \beta^{(3)}(N)+32 A_{5}(N-1)-16 A_{6}(N-1)+32 A_{8}(N-1) \\
& +8 \frac{\zeta_{2}}{N^{2}}+8 \frac{\zeta_{2}}{N}-8 \frac{\zeta_{2}}{(N+1)^{2}}-8 \frac{\zeta_{2}}{(N+1)}-16 \frac{\beta^{(1)}(N+1)}{N^{2}}-8 \frac{\beta^{(1)}(N+1)}{N} \\
& +16 \frac{\beta^{(1)}(N+2)}{(N+1)^{2}}-8 \frac{\beta^{(1)}(N+2)}{(N+1)}+8 \frac{\beta^{(2)}(N+1)}{N}-8 \frac{\beta^{(2)}(N+2)}{(N+1)}+\left(16 \frac{\beta^{(1)}(N+2)}{(N+1)}\right. \\
& +\frac{8}{(N+1)^{3}}-\frac{8}{N^{3}}-\frac{8}{N^{2}}-16 \frac{1}{(N+1)}+\frac{16}{N}+32 S_{1}(N-1) \beta^{(1)}(N) \\
& \left.-\frac{8}{(N+1)^{2}}-16 \frac{\beta^{(1)}(N+1)}{N}+32 \beta(N) \zeta_{2}-8 \beta^{(2)}(N)-32 A_{3}(N-1)\right) \ln \left(\frac{Q^{2}}{\mu^{2}}\right) \\
& +\left(-32 \beta(N) \zeta_{2}-8 \beta^{(2)}(N)+32 A_{3}(N-1)\right) S_{1}(N-1)+\left(-32 \frac{\beta^{(1)}(N+2)}{(N+1)}\right. \\
& \left.-\frac{16}{(N+1)^{3}}+\frac{16}{(N+1)^{2}}+\frac{32}{(N+1)}\right) S_{1}(N+1)+\left(32 \frac{\beta^{(1)}(N+1)}{N}+\frac{16}{N^{3}}\right. \\
& \left.+16 \frac{1}{N^{2}}-\frac{32}{N}\right) S_{1}(N)+\left(\frac{8}{(N+1)}+\frac{8}{(N+1)^{2}}\right) S_{2}(N+1)+\left(-\frac{8}{N}-\frac{8}{N^{2}}\right) S_{2}(N) \\
& \left.-48 \beta^{(1)}(N) S_{1}^{2}(N-1)-16 \beta(N) \zeta_{3}-32 \beta^{(1)}(N) \zeta_{2}\right] \\
& \Delta_{q q, C \bar{F}}^{(2)}=\Delta_{q q, D \bar{E}}^{(2)}=\Delta_{\bar{q} \bar{q}, C \bar{F}}^{(2)}=\Delta_{\bar{q} \bar{q}, D \bar{E}}^{(2)} \\
& =C_{F}\left(C_{F}-\frac{C_{A}}{2}\right)\left[\frac{14}{N^{2}}-\frac{15}{N}-\frac{12}{(N+1)^{2}}+\frac{28}{(N+1)}-\frac{13}{(N+2)}-\frac{12}{N^{3}}\right. \\
& +\frac{24}{(N+1)^{3}}+\frac{8}{N^{4}}-\frac{16}{(N+1)^{4}}+8 \frac{A_{18}(N+2)}{(N+2)}-\frac{12}{(N+2)^{3}}+\frac{8}{(N+2)^{4}}+8 \frac{\zeta_{2}}{N^{2}} \\
& -12 \frac{\zeta_{2}}{N}-16 \frac{\zeta_{2}}{(N+1)^{2}}+24 \frac{\zeta_{2}}{(N+1)}+8 \frac{\zeta_{2}}{(N+2)^{2}}-12 \frac{\zeta_{2}}{(N+2)}-16 \frac{A_{18}(N+1)}{(N+1)} \\
& +\left(-16 \frac{S_{2}(N+1)}{(N+1)}+16 \frac{\zeta_{2}}{(N+1)}\right) S_{1}(N+1)+8 \frac{A_{18}(N)}{N}+\left(-8 \frac{\zeta_{2}}{N}+8 \frac{S_{2}(N)}{N}\right) S_{1}(N) \\
& +\left(8 \frac{S_{2}(N+2)}{(N+2)}-8 \frac{\zeta_{2}}{(N+2)}\right) S_{1}(N+2)+\left(\frac{16}{(N+1)^{2}}-\frac{24}{(N+1)}\right) S_{2}(N+1) \\
& \left.+\left(\frac{12}{N}-\frac{8}{N^{2}}\right) S_{2}(N)+\left(-8 \frac{1}{(N+2)^{2}}+\frac{12}{(N+2)}\right) S_{2}(N+2)\right]
\end{aligned}
$$




$$
\begin{aligned}
& \Delta_{g g}^{(2), C_{A}}=\frac{N_{c}^{2}}{N_{c}^{2}-1}\left[\frac{4}{N^{2}}-\frac{47}{3 N}+\frac{76}{3(N+1)^{2}}-\frac{48}{(N+1)}+\frac{50}{(N+2)^{2}}\right. \\
& +\frac{191}{3(N+2)}-\frac{8}{3 N^{3}}+\frac{8}{3(N+1)^{3}}+\frac{100}{3(N+2)^{3}}+16 \frac{\beta(N+1) \zeta_{2}}{N} \\
& +32 \frac{S_{1}(N+1) \beta^{(1)}(N+2)}{(N+1)}-8 \frac{\zeta_{3}}{N}+16 \frac{\zeta_{3}}{(N+1)}-8 \frac{\zeta_{3}}{(N+2)}-16 \frac{S_{3}(N+1)}{(N+1)} \\
& +8 \frac{S_{3}(N+2)}{(N+2)}+8 \frac{S_{3}(N)}{N}-\frac{16}{3} \frac{\beta^{(1)}(N+1)}{N}-\frac{32 \beta^{(1)}(N+2)}{3(N+1)}+4 \frac{\beta^{(2)}(N+1)}{N} \\
& +8 \frac{\beta^{(2)}(N+2)}{(N+1)}+4 \frac{\beta^{(2)}(3+N)}{(N+2)}-32 \frac{A_{3}(N+1)}{(N+1)}-16 \frac{A_{3}(N+2)}{(N+2)}-16 \frac{A_{3}(N)}{N} \\
& +16 \frac{S_{1}(N+2) \beta^{(1)}(3+N)}{(N+2)}+16 \frac{S_{1}(N) \beta^{(1)}(N+1)}{N}+32 \frac{\beta(N+2) \zeta_{2}}{(N+1)}+16 \frac{\beta(3+N) \zeta_{2}}{(N+2)} \\
& \left.-\frac{16}{3} \frac{\beta^{(1)}(3+N)}{(N+2)}\right] \\
& \Delta_{g g}^{(2), C_{F}}=\frac{23}{N^{2}}-\frac{32}{N}+\frac{64}{(N+1)^{2}}-\frac{66}{(N+1)}-\frac{105}{(N+2)^{2}}+\frac{98}{(N+2)} \\
& +\left(\frac{2}{N^{2}}+\frac{8}{(N+2)^{2}}-\frac{4}{N}+\frac{12}{(N+2)}+\frac{8}{(N+1)^{2}}-\frac{8}{(N+1)}\right) \ln ^{2}\left(\frac{Q^{2}}{\mu^{2}}\right) \\
& -\frac{12}{N^{3}}-\frac{28}{(N+1)^{3}}+12 \frac{1}{N^{4}}+\frac{32}{(N+1)^{4}}-\frac{16}{(N+2)^{3}}-16 \frac{\beta(N+1) \zeta_{2}}{N} \\
& +\frac{32}{(N+2)^{4}}+\left(-\frac{32}{(N+1)}+\frac{32}{(N+1)^{2}}\right) S_{1}^{2}(N+1)+4 \frac{\zeta_{2}}{N^{2}}-8 \frac{\zeta_{2}}{N}+8 \frac{\zeta_{3}}{N} \\
& +16 \frac{\zeta_{2}}{(N+1)^{2}}-16 \frac{\zeta_{2}}{(N+1)}-16 \frac{\zeta_{3}}{(N+1)}+16 \frac{\zeta_{2}}{(N+2)^{2}}+24 \frac{\zeta_{2}}{(N+2)}+8 \frac{\zeta_{3}}{(N+2)} \\
& +16 \frac{S_{3}(N+1)}{(N+1)}-8 \frac{S_{3}(N+2)}{(N+2)}-8 \frac{S_{3}(N)}{N}+8 \frac{\beta^{(1)}(N+1)}{N^{2}}-8 \frac{\beta^{(1)}(N+1)}{N} \\
& +16 \frac{\beta^{(1)}(N+2)}{(N+1)^{2}}-8 \frac{\beta^{(1)}(N+2)}{(N+1)}+16 \frac{\beta^{(1)}(3+N)}{(N+2)^{2}}-4 \frac{\beta^{(2)}(N+1)}{N}-8 \frac{\beta^{(2)}(N+2)}{(N+1)} \\
& -4 \frac{\beta^{(2)}(3+N)}{(N+2)}+32 \frac{A_{3}(N+1)}{(N+1)}+16 \frac{A_{3}(N+2)}{(N+2)}+16 \frac{A_{3}(N)}{N}-32 \frac{\beta(N+2) \zeta_{2}}{(N+1)} \\
& -16 \frac{\beta(3+N) \zeta_{2}}{(N+2)}+\left(\frac{8}{N^{2}}-\frac{16}{N}\right) S_{1}^{2}(N)+\left(\frac{48}{(N+2)}+\frac{32}{(N+2)^{2}}\right) S_{1}^{2}(N+2)
\end{aligned}
$$




$$
\begin{aligned}
& +\left(\left(\frac{32}{(N+1)}-\frac{32}{(N+1)^{2}}\right) S_{1}(N+1)+\left(-\frac{8}{N^{2}}+\frac{16}{N}\right) S_{1}(N)+\left(-\frac{48}{(N+2)}\right.\right. \\
& \left.-\frac{32}{(N+2)^{2}}\right) S_{1}(N+2)+\frac{8}{(N+2)^{2}}+\frac{16}{(N+1)^{3}}-\frac{16}{(N+1)^{2}}+\frac{16}{(N+2)^{3}} \\
& \left.-\frac{67}{(N+2)}+\frac{4}{N^{3}}-\frac{2}{N^{2}}+\frac{7}{N}+\frac{60}{(N+1)}\right) \ln \left(\frac{Q^{2}}{\mu^{2}}\right)+\left(-32 \frac{1}{(N+1)^{3}}\right. \\
& \left.-32 \frac{\beta^{(1)}(N+2)}{(N+1)}+\frac{32}{(N+1)^{2}}-\frac{120}{(N+1)}\right) S_{1}(N+1)+\left(-\frac{4}{N^{2}}+\frac{8}{N}\right) S_{2}(N) \\
& +\left(-\frac{16}{(N+1)^{2}}+\frac{16}{(N+1)}\right) S_{2}(N+1)+\left(\frac{4}{N^{2}}-16 \frac{\beta^{(1)}(N+1)}{N}-\frac{8}{N^{3}}\right. \\
& \left.-\frac{14}{N}\right) S_{1}(N)+\left(-\frac{24}{(N+2)}-\frac{16}{(N+2)^{2}}\right) S_{2}(N+2)+\left(-\frac{16}{(N+2)^{2}}\right. \\
& \left.+\frac{134}{(N+2)}-16 \frac{\beta^{(1)}(3+N)}{(N+2)}-\frac{32}{(N+2)^{3}}\right) S_{1}(N+2) .
\end{aligned}
$$




\section{A.2 Polarized Drell-Yan Coefficient Functions}

In this appendix we present the Mellin transforms of the coefficient functions for of the polarized Drell-Yan process. We used the same notation as in Refs. [5].

$$
\begin{aligned}
& \delta \Delta_{a b}=\delta \Delta_{a b}^{(0)}+a_{s} \delta \Delta_{a b}^{(1)}+a_{s}^{2} \delta \Delta_{a b}^{(2)} \\
& \delta \Delta_{q \bar{q}}^{(0)}=-\Delta_{q \bar{q}}^{(0)} \\
& \delta \Delta_{q \bar{q}}^{(1)}=-\Delta_{q \bar{q}}^{(1)} \\
& \delta \Delta_{q \bar{q}}^{(2), S+V}=-\Delta_{q \bar{q}}^{(2), S+V} \\
& \delta \Delta_{q \bar{q}}^{(2), C_{A}}=-\Delta_{q \bar{q}}^{(2), C_{A}} \\
& \delta \Delta_{q \bar{q}}^{(2), C_{F}}=-\Delta_{q \bar{q}}^{(2), C_{F}} \\
& \delta \Delta_{q \bar{q}, A \bar{A}}^{(2)}=-\Delta_{q \bar{q}, A \bar{A}}^{(2)} \\
& \delta \Delta_{q \bar{q}, A \bar{C}}^{(2)}=\delta \Delta_{q \bar{q}, A \bar{D}}^{(2)}=-\Delta_{q \bar{q}, A \bar{D}}^{(2)} \\
& \delta \Delta_{q \bar{q}, B \bar{B}}^{(2)}==-\Delta_{q \bar{q}, B \bar{B}}^{(2)} \\
& \delta \Delta_{q \bar{q}, B \bar{C}}^{(2)}=\delta \Delta_{q \bar{q}, B \bar{D}}^{(2)}=-\Delta_{q \bar{q}, B \bar{C}}^{(2)} \\
& \delta \Delta_{q g}^{(2), C_{A}}=C_{A} T_{f}\left[\frac{144}{N^{2}}-\frac{144}{N}-\frac{140}{(N+1)^{2}}+\frac{142}{(N+1)}+\frac{6}{(N+2)}\right. \\
& -\frac{94}{N^{3}}+\frac{184}{(N+1)^{3}}+\frac{44}{N^{4}}+\frac{64}{(N+1)^{4}}+\frac{24}{(N+2)^{3}}+44 \frac{\zeta_{2}}{N^{2}}-96 \frac{\zeta_{2}}{N} \\
& -18 \frac{\zeta_{3}}{N}+32 \frac{\zeta_{2}}{(N+1)^{2}}+68 \frac{\zeta_{2}}{(N+1)}+36 \frac{\zeta_{3}}{(N+1)}+24 \frac{\zeta_{2}}{(N+2)}-36 \frac{S_{1}(N+2)}{(N+2)^{2}} \\
& -\frac{16}{3} \frac{S_{3}(N+1)}{(N+1)}+\frac{8}{3} \frac{S_{3}(N)}{N}-8 \frac{\beta^{(1)}(N+1)}{N^{2}}+24 \frac{\beta^{(1)}(N+1)}{N}-16 \frac{\beta^{(1)}(N+2)}{(N+1)^{2}} \\
& +24 \frac{\beta^{(1)}(N+2)}{(N+1)}+4 \frac{\beta^{(2)}(N+1)}{N}+8 \frac{\beta^{(2)}(N+2)}{(N+1)}+12 \frac{S_{1}^{2}(N+2)}{(N+2)}+\left(-\frac{48}{(N+1)^{3}}\right. \\
& -\frac{176}{(N+1)^{2}}-52 \frac{S_{2}(N+1)}{(N+1)}+32 \frac{\beta^{(1)}(N+2)}{(N+1)}+\frac{142}{(N+1)} \\
& \left.+56 \frac{\zeta_{2}}{(N+1)}\right) S_{1}(N+1)-24 \frac{A_{18}(N+1)}{(N+1)}+12 \frac{A_{18}(N)}{N}+\left(-\frac{136}{N}-28 \frac{\zeta_{2}}{N}-\frac{56}{N^{3}}\right.
\end{aligned}
$$




$$
\begin{aligned}
& \left.+26 \frac{S_{2}(N)}{N}+16 \frac{\beta^{(1)}(N+1)}{N}+\frac{108}{N^{2}}\right) S_{1}(N)+\left(\frac{120}{N}-\frac{44}{N^{2}}\right) S_{2}(N)+\left(\frac{8}{(N+1)^{2}}\right. \\
& \left.+\frac{92}{(N+1)}\right) S_{1}^{2}(N+1)+\left(-\frac{104}{N}+\frac{44}{N^{2}}\right) S_{1}^{2}(N)+\left(-4 \frac{S_{1}(N)}{N}+\frac{8}{N^{2}}-\frac{24}{N}\right. \\
& \left.+\frac{24}{(N+1)}+\frac{8}{(N+1)^{2}}+8 \frac{S_{1}(N+1)}{(N+1)}\right) \ln ^{2}\left(\frac{Q^{2}}{\mu^{2}}\right)+\left(\left(-\frac{16}{(N+1)^{2}}\right.\right. \\
& \left.-\frac{88}{(N+1)}\right) S_{1}(N+1)+\left(\frac{100}{N}-\frac{40}{N^{2}}\right) S_{1}(N)-24 \frac{S_{1}^{2}(N+1)}{(N+1)}+\frac{88}{(N+1)^{2}} \\
& +8 \frac{S_{2}(N+1)}{(N+1)}+\frac{24}{N^{3}}+4 \frac{\zeta_{2}}{N}-\frac{72}{(N+1)}-4 \frac{S_{2}(N)}{N}-12 \frac{S_{1}(N+2)}{(N+2)}+\frac{12}{(N+2)^{2}} \\
& -\frac{52}{N^{2}}+12 \frac{S_{1}^{2}(N)}{N}-8 \frac{\beta^{(1)}(N+1)}{N}+\frac{68}{N}-8 \frac{\zeta_{2}}{(N+1)}+\frac{32}{(N+1)^{3}} \\
& \left.-16 \frac{\beta^{(1)}(N+2)}{(N+1)}\right) \ln \left(\frac{Q^{2}}{\mu^{2}}\right)+\left(-\frac{116}{(N+1)}-\frac{32}{(N+1)^{2}}\right) S_{2}(N+1)-\frac{26 S_{1}^{3}(N)}{3 N} \\
& \left.+\frac{52 S_{1}^{3}(N+1)}{3(N+1)}\right] \\
& \delta \Delta_{q g}^{(2), C_{F}}=C_{F} T_{f}\left[\frac{175}{N^{2}}-\frac{627}{2 N}-\frac{79}{(N+1)^{2}}+\frac{331}{(N+1)}-\frac{60}{(N+2)^{2}}\right. \\
& -\frac{15}{2(N+2)}+\left(\frac{104}{(N+1)}-\frac{84}{(N+1)^{2}}\right) S_{1}^{2}(N+1)-\frac{81}{N^{3}}-\frac{40}{(N+1)^{3}} \\
& +\frac{34}{N^{4}}-\frac{68}{(N+1)^{4}}+\left(-56 \frac{\zeta_{2}}{N}+2 \frac{S_{2}(N)}{N}-\frac{48}{N^{3}}+\frac{140}{N^{2}}-\frac{256}{N}\right) S_{1}(N) \\
& +\frac{12}{(N+2)^{3}}+\left(\frac{42}{N^{2}}-\frac{110}{N}\right) S_{1}^{2}(N)+28 \frac{\zeta_{2}}{N^{2}}-126 \frac{\zeta_{2}}{N}+32 \frac{\zeta_{3}}{N}-56 \frac{\zeta_{2}}{(N+1)^{2}} \\
& +128 \frac{\zeta_{2}}{(N+1)}-64 \frac{\zeta_{3}}{(N+1)}+4 \frac{S_{2}(N+1)}{(N+1)^{2}}-6 \frac{S_{2}(N+2)}{(N+2)}+\frac{16}{3} \frac{S_{3}(N+1)}{(N+1)} \\
& -\frac{8}{3} \frac{S_{3}(N)}{N}+16 \frac{\beta^{(1)}(N+1)}{N^{2}}-32 \frac{\beta^{(1)}(N+1)}{N}-32 \frac{\beta^{(1)}(N+2)}{(N+1)^{2}}-32 \frac{\beta^{(1)}(N+2)}{(N+1)} \\
& +18 \frac{S_{1}^{2}(N+2)}{(N+2)}+32 \frac{A_{18}(N+1)}{(N+1)}-16 \frac{A_{18}(N)}{N}+\left(-4 \frac{S_{2}(N+1)}{(N+1)}+\frac{154}{(N+1)}\right.
\end{aligned}
$$




$$
\begin{aligned}
& \left.+112 \frac{\zeta_{2}}{(N+1)}-\frac{48}{(N+1)^{2}}+\frac{96}{(N+1)^{3}}\right) S_{1}(N+1)+\left(\left(\frac{80}{(N+1)^{2}}\right.\right. \\
& \left.-\frac{72}{(N+1)}\right) S_{1}(N+1)+\left(\frac{96}{N}-\frac{40}{N^{2}}\right) S_{1}(N)+\frac{16}{N^{3}}+36 \frac{S_{1}^{2}(N)}{N} \\
& -72 \frac{S_{1}^{2}(N+1)}{(N+1)}+32 \frac{\zeta_{2}}{N}-\frac{76}{(N+1)}-\frac{32}{(N+1)^{3}}-\frac{58}{N^{2}}-4 \frac{S_{2}(N)}{N} \\
& +8 \frac{S_{2}(N+1)}{(N+1)}-12 \frac{S_{1}(N+2)}{(N+2)}+\frac{98}{N}+\frac{12}{(N+2)^{2}}+\frac{16}{(N+1)^{2}} \\
& \left.-64 \frac{\zeta_{2}}{(N+1)}\right) \ln \left(\frac{Q^{2}}{\mu^{2}}\right)+\left(\frac{4}{N}-\frac{2}{N^{2}}\right) S_{2}(N)+\left(\frac{60}{(N+2)}\right. \\
& \left.-\frac{24}{(N+2)^{2}}\right) S_{1}(N+2)+\left(\frac{6}{N^{2}}-12 \frac{S_{1}(N)}{N}-\frac{9}{N}-\frac{12}{(N+1)^{2}}\right. \\
& \left.\left.+24 \frac{S_{1}(N+1)}{(N+1)}\right) \ln ^{2}\left(\frac{Q^{2}}{\mu^{2}}\right)-\frac{70 S_{1}^{3}(N)}{3 N}+\frac{140 S_{1}^{3}(N+1)}{3(N+1)}\right] \\
& \delta \Delta_{q \bar{q}, C \bar{C}}^{(2)}=\delta \Delta_{q \bar{q}, D \bar{D}}^{(2)}=\delta \Delta_{q q, C \bar{C}}^{(2)}=\delta \Delta_{q q, D \bar{D}}^{(2)}=\delta \Delta_{\bar{q}, C \bar{C}}^{(2)}=\delta \Delta_{\bar{q} \bar{q}, D \bar{D}}^{(2)} \\
& =C_{F} T_{f}\left[\left(\frac{10}{(N+1)}-\frac{10}{N}+\frac{4}{N^{2}}+\frac{4}{(N+1)^{2}}\right) \ln ^{2}\left(\frac{Q^{2}}{\mu^{2}}\right)+\left(\left(-\frac{40}{(N+1)}\right.\right.\right. \\
& \left.-\frac{16}{(N+1)^{2}}\right) S_{1}(N+1)+\left(-\frac{16}{N^{2}}+\frac{40}{N}\right) S_{1}(N)+\frac{16}{(N+1)^{3}}-\frac{40}{(N+1)} \\
& \left.+\frac{40}{N}-\frac{28}{N^{2}}+\frac{32}{(N+1)^{2}}+\frac{16}{N^{3}}\right) \ln \left(\frac{Q^{2}}{\mu^{2}}\right)+\left(-\frac{32}{(N+1)^{3}}-\frac{64}{(N+1)^{2}}\right. \\
& \left.+\frac{80}{(N+1)}\right) S_{1}(N+1)+\left(-\frac{80}{N}+\frac{56}{N^{2}}-\frac{32}{N^{3}}\right) S_{1}(N)+\left(\frac{16}{(N+1)^{2}}\right. \\
& \left.+40 \frac{1}{(N+1)}\right) S_{1}^{2}(N+1)+\left(\frac{16}{N^{2}}-\frac{40}{N}\right) S_{1}^{2}(N)+\left(-\frac{24}{(N+1)^{2}}\right. \\
& \left.-\frac{52}{(N+1)}\right) S_{2}(N+1)+\left(\frac{52}{N}-\frac{24}{N^{2}}\right) S_{2}(N)+52 \frac{\zeta_{2}}{(N+1)}+24 \frac{\zeta_{2}}{(N+1)^{2}} \\
& +\frac{84}{N^{2}}-\frac{131}{N}-\frac{50}{N^{3}}+\frac{36}{N^{4}}-52 \frac{\zeta_{2}}{N}+\frac{78}{(N+1)^{3}}-\frac{34}{(N+1)^{2}} \\
& \left.+\frac{36}{(N+1)^{4}}+\frac{128}{(N+1)}+24 \frac{\zeta_{2}}{N^{2}}+\frac{3}{(N+2)}\right]
\end{aligned}
$$




$$
\begin{aligned}
& \delta \Delta_{q \bar{q}, C \bar{D}}^{(2)}=-\delta \Delta_{q q, C \bar{D}}^{(2)}=-\delta \Delta_{\bar{q} \bar{q}, C \bar{D}}^{(2)} \\
& =C_{F} T_{f}\left[-\frac{16}{N^{2}}+\frac{32}{N}-\frac{32}{(N+1)}+\frac{8}{(N+1)^{3}}-\frac{32}{(N+1)^{4}}\right. \\
& +192 \frac{\beta(N+1) \zeta_{2}}{N}+16 \frac{\zeta_{2}}{N^{2}}-8 \frac{\zeta_{2}}{N}-16 \frac{\zeta_{3}}{N}+24 \frac{\zeta_{2}}{(N+1)^{2}}+8 \frac{\zeta_{3}}{(N+1)}-24 \frac{S_{2}(N+1)}{(N+1)^{2}} \\
& -8 \frac{S_{3}(N+1)}{(N+1)}+16 \frac{S_{3}(N)}{N}-32 \frac{\beta^{(1)}(N+1)}{N^{2}}+16 \frac{\beta^{(1)}(N+1)}{N}-80 \frac{\beta^{(1)}(N+2)}{(N+1)^{2}} \\
& +16 \frac{\beta^{(1)}(N+2)}{(N+1)}-16 \frac{\beta^{(2)}(N+1)}{N}-8 \frac{\beta^{(2)}(N+2)}{(N+1)}-96 \frac{A_{3}(N+1)}{(N+1)}-192 \frac{A_{3}(N)}{N} \\
& +96 \frac{\beta(N+2) \zeta_{2}}{(N+1)}+8 \frac{A_{18}(N+1)}{(N+1)}-16 \frac{A_{18}(N)}{N}+\left(-\frac{16}{N^{2}}+\frac{8}{N}\right) S_{2}(N)+\left(16 \frac{\zeta_{2}}{N}\right. \\
& \left.+192 \frac{\beta^{(1)}(N+1)}{N}-16 \frac{S_{2}(N)}{N}\right) S_{1}(N)+\left(96 \frac{\beta^{(1)}(N+2)}{(N+1)}+8 \frac{S_{2}(N+1)}{(N+1)}\right. \\
& \left.\left.-8 \frac{\zeta_{2}}{(N+1)}\right) S_{1}(N+1)\right] \\
& \delta \Delta_{g g}^{(2), C_{A}}=\frac{N_{c}^{2}}{N_{c}^{2}-1}\left[-\frac{4}{N^{2}}+\frac{101}{3 N}-\frac{124}{3(N+1)^{2}}-\frac{22}{(N+2)^{2}}-\frac{101}{3(N+2)}\right. \\
& +\frac{8}{3 N^{3}}-\frac{56}{3(N+1)^{3}}-\frac{28}{3(N+2)^{3}}-16 \frac{\beta(N+1) \zeta_{2}}{N}-32 \frac{S_{1}(N+1) \beta^{(1)}(N+2)}{(N+1)} \\
& -8 \frac{\zeta_{3}}{N}+16 \frac{\zeta_{3}}{(N+1)}-8 \frac{\zeta_{3}}{(N+2)}-16 \frac{S_{3}(N+1)}{(N+1)}+8 \frac{S_{3}(N+2)}{(N+2)}+8 \frac{S_{3}(N)}{N} \\
& +\frac{16}{3} \frac{\beta^{(1)}(N+1)}{N}+\frac{32 \beta^{(1)}(N+2)}{3(N+1)}-4 \frac{\beta^{(2)}(N+1)}{N}-8 \frac{\beta^{(2)}(N+2)}{(N+1)}-4 \frac{\beta^{(2)}(3+N)}{(N+2)} \\
& +32 \frac{A_{3}(N+1)}{(N+1)}+16 \frac{A_{3}(N+2)}{(N+2)}+16 \frac{A_{3}(N)}{N}-16 \frac{S_{1}(N+2) \beta^{(1)}(3+N)}{(N+2)} \\
& \left.-16 \frac{S_{1}(N) \beta^{(1)}(N+1)}{N}-32 \frac{\beta(N+2) \zeta_{2}}{(N+1)}-16 \frac{\beta(3+N) \zeta_{2}}{(N+2)}+\frac{16}{3} \frac{\beta^{(1)}(3+N)}{(N+2)}\right] \\
& \delta \Delta_{q q, C \bar{E}}^{(2)}=\delta \Delta_{q q, D \bar{F}}^{(2)}=\delta \Delta_{\overline{q q}, C \bar{E}}^{(2)}=\delta \Delta_{\overline{q q}, D \bar{F}}^{(2)}=\Delta_{q q, C \bar{E}}^{(2)} \\
& \delta \Delta_{q q, C \bar{F}}^{(2)}=\delta \Delta_{q q, D \bar{E}}^{(2)}=\delta \Delta_{\overline{q q}, C \bar{F}}^{(2)}=\delta \Delta_{\overline{q q}, D \bar{E}}^{(2)}=\Delta_{q q, C \bar{F}}^{(2)}
\end{aligned}
$$




$$
\begin{aligned}
& \delta \Delta_{g g}^{(2), C_{F}}=-\frac{55}{N^{2}}+\frac{70}{N}+\frac{72}{(N+1)^{2}}-\frac{62}{(N+1)}-\frac{27}{(N+2)^{2}}-\frac{8}{(N+2)} \\
& +\frac{28}{N^{3}}+\frac{12}{(N+1)^{3}}-\frac{12}{N^{4}}-\frac{32}{(N+1)^{4}}+16 \frac{\beta(N+1) \zeta_{2}}{N}-\frac{16}{(N+2)^{4}} \\
& +\left(16 \frac{1}{(N+1)^{2}}+\frac{16}{(N+1)}\right) S_{2}(N+1)+\left(\frac{4}{N^{2}}-\frac{16}{N}\right) S_{2}(N)+\left(-\frac{2}{N^{2}}-\frac{8}{(N+1)^{2}}\right. \\
& \left.+\frac{8}{N}-\frac{8}{(N+1)}\right) \ln ^{2}\left(\frac{Q^{2}}{\mu^{2}}\right)-4 \frac{\zeta_{2}}{N^{2}}+16 \frac{\zeta_{2}}{N}+8 \frac{\zeta_{3}}{N}-16 \frac{\zeta_{2}}{(N+1)^{2}}-16 \frac{\zeta_{2}}{(N+1)} \\
& -16 \frac{\zeta_{3}}{(N+1)}+8 \frac{\zeta_{3}}{(N+2)}+16 \frac{S_{3}(N+1)}{(N+1)}-8 \frac{S_{3}(N+2)}{(N+2)}-8 \frac{S_{3}(N)}{N}-8 \frac{\beta^{(1)}(N+1)}{N^{2}} \\
& +8 \frac{\beta^{(1)}(N+1)}{N}-16 \frac{\beta^{(1)}(N+2)}{(N+1)^{2}}+8 \frac{\beta^{(1)}(N+2)}{(N+1)}-16 \frac{\beta^{(1)}(3+N)}{(N+2)^{2}}+4 \frac{\beta^{(2)}(N+1)}{N} \\
& +8 \frac{\beta^{(2)}(N+2)}{(N+1)}+4 \frac{\beta^{(2)}(3+N)}{(N+2)}-32 \frac{A_{3}(N+1)}{(N+1)}-16 \frac{A_{3}(N+2)}{(N+2)}-16 \frac{A_{3}(N)}{N} \\
& +32 \frac{\beta(N+2) \zeta_{2}}{(N+1)}+16 \frac{\beta(3+N) \zeta_{2}}{(N+2)}+\left(\frac{86}{N}+\frac{8}{N^{3}}-\frac{36}{N^{2}}+16 \frac{\beta^{(1)}(N+1)}{N}\right) S_{1}(N) \\
& +\left(16 \frac{\beta^{(1)}(3+N)}{(N+2)}+\frac{18}{(N+2)}\right) S_{1}(N+2)+\left(-\frac{32}{(N+1)^{2}}-\frac{32}{(N+1)}\right) S_{1}^{2}(N+1) \\
& +\left(-\frac{8}{N^{2}}+\frac{32}{N}\right) S_{1}^{2}(N)+\left(\left(\frac{32}{(N+1)}+\frac{32}{(N+1)^{2}}\right) S_{1}(N+1)+\left(\frac{8}{N^{2}}\right.\right. \\
& \left.-\frac{32}{N}\right) S_{1}(N)-\frac{16}{(N+1)^{2}}-\frac{16}{(N+1)^{3}}+\frac{18}{N^{2}}+\frac{52}{(N+1)}-\frac{4}{N^{3}}-\frac{43}{N} \\
& \left.-\frac{9}{(N+2)}\right) \ln \left(\frac{Q^{2}}{\mu^{2}}\right)+\left(\frac{32}{(N+1)^{3}}+\frac{32}{(N+1)^{2}}-\frac{104}{(N+1)}\right. \\
& \left.+32 \frac{\beta^{(1)}(N+2)}{(N+1)}\right) S_{1}(N+1) .
\end{aligned}
$$




\section{A.3. Scalar Higgs Coefficient Functions}

In this appendix we present the Mellin transforms for the coefficient functions of the hadronic Higgs-production cross section in the heavy-mass limit. We used the same notation as in Refs. [6].

$$
\begin{aligned}
& \Delta_{a b}=\Delta_{a b}^{(0)}+a_{s} \Delta_{a b}^{(1)}+a_{s}^{2} \Delta_{a b}^{(2)} \\
& \Delta_{g g}^{(0)}=1 \\
& \Delta_{g g}^{(1)}=C_{A}\left[\left(-\frac{8}{(N+3)}-8 S_{1}(N-1)+\frac{8}{(N+2)}-\frac{16}{(N+1)}\right) \ln \left(\frac{Q^{2}}{\mu^{2}}\right)-\frac{22}{3 N}\right. \\
& -\frac{16}{(N+1)^{2}}+8 S_{1}^{2}(N-1)+\frac{8}{(N+2)^{2}}+\frac{22}{3(N+3)}-\frac{22}{(N+2)}+\frac{22}{(N+1)} \\
& \left.+32 \frac{S_{1}(N+1)}{(N+1)}+16 \zeta_{2}-\frac{8}{(N+3)^{2}}-16 \frac{S_{1}(N+2)}{(N+2)}+16 \frac{S_{1}(N+3)}{(N+3)}\right] \\
& \Delta_{g q}^{(1)}=C_{F}\left[\left(-\frac{4}{(N+1)}+\frac{4}{N}+\frac{2}{(N+2)}\right) \ln \left(\frac{Q^{2}}{\mu^{2}}\right)+\frac{4}{N^{2}}+\frac{6}{(N+1)}-\frac{3}{N}\right. \\
& \left.-\frac{4}{(N+1)^{2}}-\frac{1}{(N+2)}+8 \frac{S_{1}(N+1)}{(N+1)}+\frac{2}{(N+2)^{2}}-8 \frac{S_{1}(N)}{N}-4 \frac{S_{1}(N+2)}{(N+2)}\right] \\
& \Delta_{q \bar{q}}^{(1)}=C_{F}^{2}\left[\frac{8}{3 N}-\frac{8}{(N+1)}+\frac{8}{(N+2)}-\frac{8}{3(N+3)}\right] \\
& \Delta_{g g}^{(2), C_{A}^{2}}=93+\frac{1072}{9} \zeta_{2}+\left(\frac{96}{(N+2)^{2}}+\frac{132}{(N+3)}-\frac{32}{(N+3)^{2}}-\frac{352}{3 N}\right. \\
& -64 \frac{S_{1}(N+2)}{(N+2)}+32 S_{1}^{2}(N-1)+64 \frac{S_{1}(N+3)}{(N+3)}-\frac{332}{3(N+2)}+\frac{44}{3} S_{1}(N-1) \\
& \left.+128 \frac{S_{1}(N+1)}{(N+1)}+\frac{376}{3(N+1)}\right) \ln ^{2}\left(\frac{Q^{2}}{\mu^{2}}\right)-\frac{16705}{27 N}-\frac{2188}{3(N+1)^{2}} \\
& +\frac{18523}{27(N+1)}+\frac{1582}{3(N+2)^{2}}-\frac{19751}{27(N+2)}-\frac{8108}{9(N+3)^{2}}+\frac{7055}{9(N+3)} \\
& +\frac{3602}{9 N^{2}}-\frac{16}{N^{4}}-\frac{572}{3 N^{3}}-\frac{32}{(N+1)^{4}}+\frac{328}{(N+1)^{3}}+\frac{272}{(N+2)^{4}} \\
& -\frac{604}{(N+2)^{3}}-\frac{144}{(N+3)^{4}}+\frac{1496}{3(N+3)^{3}}-\frac{44}{3} \zeta_{3}-\frac{176}{9} S_{3}(N-1)
\end{aligned}
$$




$$
\begin{aligned}
& -\frac{44}{3} A_{18}(N-1)-8 \frac{\zeta_{2}}{N^{2}}-\frac{16}{3} \beta^{(3)}(N)-32 A_{5}(N-1)+16 A_{6}(N-1) \\
& -32 A_{8}(N-1)-8 A_{21}(N-1)+12 A_{22}(N-1)-38 S_{4}(N-1) \\
& +\frac{176}{9} S_{1}^{3}(N-1)+32 S_{1}^{4}(N-1)-2 S_{2}^{2}(N-1)-356 \frac{\zeta_{2}}{N}-16 \frac{\zeta_{3}}{N}-168 \frac{\zeta_{2}}{(N+1)^{2}} \\
& +500 \frac{\zeta_{2}}{(N+1)}-80 \frac{\zeta_{3}}{(N+1)}+296 \frac{\zeta_{2}}{(N+2)^{2}}-456 \frac{\zeta_{2}}{(N+2)}+16 \frac{\zeta_{3}}{(N+2)}-152 \frac{\zeta_{2}}{(N+3)^{2}} \\
& +\frac{968 \zeta_{2}}{3(N+3)}-32 \frac{\zeta_{3}}{(N+3)}+20 S_{2}(N-1) \zeta_{2}-32 \frac{S_{3}(N+1)}{(N+1)}+40 \frac{S_{3}(N+2)}{(N+2)} \\
& -24 \frac{S_{3}(N+3)}{(N+3)}+16 \frac{S_{3}(N)}{N}+16 \beta(N) \zeta_{3}+16 \frac{\beta^{(1)}(N+1)}{N^{2}}+\frac{112 \beta^{(1)}(N+1)}{3 N} \\
& -80 \frac{\beta^{(1)}(N+2)}{(N+1)^{2}}+32 \frac{\beta^{(1)}(N+2)}{(N+1)}-16 \frac{\beta^{(1)}(N+3)}{(N+2)^{2}}-56 \frac{\beta^{(1)}(N+3)}{(N+2)}-48 \frac{\beta^{(1)}(4+N)}{(N+3)^{2}} \\
& -\frac{88 \beta^{(1)}(4+N)}{3(N+3)}+32 \beta^{(1)}(N) \zeta_{2}+4 \frac{\beta^{(2)}(N+1)}{N}+20 \frac{\beta^{(2)}(N+2)}{(N+1)}+16 \frac{\beta^{(2)}(N+3)}{(N+2)} \\
& +16 \frac{\beta^{(2)}(4+N)}{(N+3)}-16 \frac{A_{3}(N+1)}{(N+1)}+64 \frac{A_{3}(N+2)}{(N+2)}+64 \frac{A_{3}(N+3)}{(N+3)}+48 \frac{A_{3}(N)}{N} \\
& +32 \frac{A_{18}(N+1)}{(N+1)}-40 \frac{A_{18}(N+2)}{(N+2)}+24 \frac{A_{18}(N+3)}{(N+3)}-16 \frac{A_{18}(N)}{N}-528 \frac{S_{1}^{2}(N)}{N} \\
& +256 \frac{S_{1}^{3}(N+1)}{(N+1)}-128 \frac{S_{1}^{3}(N+2)}{(N+2)}+128 \frac{S_{1}^{3}(N+3)}{(N+3)}+\frac{446}{5} \zeta_{2}^{2}-48 \frac{\beta(N+1) \zeta_{2}}{N} \\
& +16 \frac{\beta(N+2) \zeta_{2}}{(N+1)}-64 \frac{\beta(N+3) \zeta_{2}}{(N+2)}-64 \frac{\beta(4+N) \zeta_{2}}{(N+3)}+\left(\frac{2848}{3(N+2)^{2}}+72 \frac{S_{2}(N+2)}{(N+2)}\right. \\
& \left.-296 \frac{\zeta_{2}}{(N+2)}-\frac{7576}{9(N+2)}-\frac{608}{(N+2)^{3}}\right) S_{1}(N+2)+\left(-48 \frac{\beta^{(1)}(N+1)}{N}\right. \\
& \left.-\frac{8108}{9 N}-16 \frac{S_{2}(N)}{N}+\frac{1232}{3 N^{2}}+16 \frac{\zeta_{2}}{N}\right) S_{1}(N)+\left(\frac{8}{N^{2}}+\frac{760}{3 N}\right) S_{2}(N) \\
& +\left(\frac{48}{(N+3)^{2}}-\frac{220}{(N+3)}\right) S_{2}(N+3)+\left(-52 S_{2}(N-1)+48 \beta^{(1)}(N)+164 \zeta_{2}\right. \\
& \left.+\frac{536}{9}\right) S_{1}^{2}(N-1)+\left(\frac{2032}{3(N+1)}-\frac{240}{(N+1)^{2}}\right) S_{1}^{2}(N+1)+\left(\frac{504}{(N+2)^{2}}\right.
\end{aligned}
$$




$$
\begin{aligned}
& \left.-\frac{1856}{3(N+2)}\right) S_{1}^{2}(N+2)+\left(-\frac{248}{(N+3)^{2}}+\frac{1760}{3(N+3)}\right) S_{1}^{2}(N+3)+(-24 \\
& -\frac{176}{3} \zeta_{2}+\left(\frac{1856}{3(N+2)}-\frac{480}{(N+2)^{2}}\right) S_{1}(N+2)+\frac{1262}{3 N}+\frac{904}{3(N+1)^{2}} \\
& -\frac{3562}{9(N+1)}-\frac{1328}{3(N+2)^{2}}+\frac{3026}{9(N+2)}+\frac{1496}{3(N+3)^{2}}-\frac{4322}{9(N+3)} \\
& -\frac{88}{3} S_{1}^{2}(N-1)-\frac{176}{N^{2}}+\frac{256}{(N+2)^{3}}-\frac{96}{(N+3)^{3}}+8 \zeta_{3}+16 S_{3}(N-1) \\
& +8 \beta^{(2)}(N)+32 A_{3}(N-1)-64 S_{1}^{3}(N-1)-288 \frac{\zeta_{2}}{(N+1)}+144 \frac{\zeta_{2}}{(N+2)} \\
& -144 \frac{\zeta_{2}}{(N+3)}+528 \frac{S_{1}(N)}{N}+64 \frac{S_{2}(N+1)}{(N+1)}-32 \frac{S_{2}(N+2)}{(N+2)}+32 \frac{S_{2}(N+3)}{(N+3)} \\
& -64 \frac{\beta^{(1)}(N+2)}{(N+1)}-32 \frac{\beta^{(1)}(N+3)}{(N+2)}-32 \frac{\beta^{(1)}(4+N)}{(N+3)}-384 \frac{S_{1}^{2}(N+1)}{(N+1)} \\
& +192 \frac{S_{1}^{2}(N+2)}{(N+2)}-192 \frac{S_{1}^{2}(N+3)}{(N+3)}+\left(-144 \zeta_{2}-\frac{536}{9}-32 \beta^{(1)}(N)\right. \\
& \left.+32 S_{2}(N-1)\right) S_{1}(N-1)+\left(\frac{224}{(N+3)^{2}}-\frac{1760}{3(N+3)}\right) S_{1}(N+3) \\
& \left.+\left(\frac{192}{(N+1)^{2}}-\frac{2032}{3(N+1)}\right) S_{1}(N+1)-32 \beta(N) \zeta_{2}\right) \ln \left(\frac{Q^{2}}{\mu^{2}}\right)+\left(-\frac{44}{3} S_{2}(N-1)\right. \\
& +32 \beta(N) \zeta_{2}-48 \zeta_{3}-8 S_{3}(N-1)+8 A_{18}(N-1)+8 \beta^{(2)}(N)+\frac{1616}{27} \\
& \left.-32 A_{3}(N-1)+\frac{44}{3} \zeta_{2}\right) S_{1}(N-1)+\left(-\frac{40}{(N+1)^{2}}-\frac{192}{(N+1)}\right) S_{2}(N+1) \\
& +\left(-\frac{2992}{3(N+3)^{2}}+\frac{288}{(N+3)^{3}}-88 \frac{S_{2}(N+3)}{(N+3)}+\frac{1020}{(N+3)}\right. \\
& \left.+312 \frac{\zeta_{2}}{(N+3)}\right) S_{1}(N+3)+\left(144 \frac{\beta^{(1)}(N+2)}{(N+1)}-192 \frac{S_{2}(N+1)}{(N+1)}+640 \frac{\zeta_{2}}{(N+1)}\right. \\
& \left.+\frac{192}{(N+1)^{3}}-\frac{2144}{3(N+1)^{2}}+\frac{8636}{9(N+1)}\right) S_{1}(N+1)+\left(-\frac{192}{(N+2)^{2}}\right. \\
& \left.+\frac{148}{(N+2)}\right) S_{2}(N+2)
\end{aligned}
$$




$$
\begin{aligned}
& \Delta_{g g}^{(2), C_{A} T_{f} n_{f}}=-\frac{320}{9} \zeta_{2}+\frac{3142}{27 N}+\frac{208}{3(N+1)^{2}}-\frac{6188}{27(N+1)}-\frac{268}{3(N+2)^{2}} \\
& +\frac{5218}{27(N+2)}+\frac{64}{(N+3)^{2}}-\frac{3068}{27(N+3)}-\frac{160}{9} S_{1}^{2}(N-1)-\frac{296}{9 N^{2}} \\
& +\frac{16}{3 N^{3}}-\frac{16}{(N+1)^{3}}+\frac{40}{(N+2)^{3}}-\frac{32}{3(N+3)^{3}}-\frac{80}{3} \zeta_{3}+\frac{64}{9} S_{3}(N-1) \\
& +\frac{16}{3} A_{18}(N-1)-\frac{64}{9} S_{1}^{3}(N-1)+\frac{16 \zeta_{2}}{3 N}-32 \frac{\zeta_{2}}{(N+1)}+24 \frac{\zeta_{2}}{(N+2)}-\frac{32 \zeta_{2}}{3(N+3)} \\
& +32 \frac{S_{2}(N+1)}{(N+1)}-24 \frac{S_{2}(N+2)}{(N+2)}+\frac{32 S_{2}(N+3)}{3(N+3)}-\frac{16 S_{2}(N)}{3 N}+16 \frac{A_{18}(N+1)}{(N+1)} \\
& -8 \frac{A_{18}(N+2)}{(N+2)}-16 \frac{A_{18}(N)}{N}-\frac{128 S_{1}^{2}(N+1)}{3(N+1)}+\frac{64 S_{1}^{2}(N+2)}{3(N+2)}-\frac{64 S_{1}^{2}(N+3)}{3(N+3)} \\
& -\frac{160}{3}+\left(\frac{64}{3(N+3)^{2}}-\frac{304}{3(N+3)}\right) S_{1}(N+3)+\left(-16 \frac{S_{2}(N)}{N}+16 \frac{\zeta_{2}}{N}+\frac{592}{9 N}\right) S_{1}(N) \\
& +\left(\frac{128 S_{1}(N+1)}{3(N+1)}+\frac{64}{3(N+2)^{2}}-\frac{568}{9(N+2)}+\frac{32}{3} S_{1}^{2}(N-1)+16-\frac{32}{3(N+1)^{2}}\right. \\
& +\frac{728}{9(N+1)}-\frac{64 S_{1}(N+2)}{3(N+2)}+\frac{152}{3(N+3)}+\frac{64}{3} \zeta_{2}+\frac{160}{9} S_{1}(N-1)-\frac{296}{9 N} \\
& \left.-\frac{32}{3(N+3)^{2}}+\frac{64 S_{1}(N+3)}{3(N+3)}\right) \ln \left(\frac{Q^{2}}{\mu^{2}}\right)+\left(-\frac{16}{3} \zeta_{2}-\frac{448}{27}+\frac{16}{3} S_{2}(N-1)\right) S_{1}(N-1) \\
& +\left(16 \frac{S_{2}(N+1)}{(N+1)}+\frac{64}{3(N+1)^{2}}-\frac{1456}{9(N+1)}-16 \frac{\zeta_{2}}{(N+1)}\right) S_{1}(N+1)+\left(8 \frac{\zeta_{2}}{(N+2)}\right. \\
& \left.+\frac{1160}{9(N+2)}-8 \frac{S_{2}(N+2)}{(N+2)}-\frac{128}{3(N+2)^{2}}\right) S_{1}(N+2)+\left(-\frac{16}{3} S_{1}(N-1)\right. \\
& \left.+\frac{16}{3(N+2)}-\frac{32}{3(N+1)}-\frac{16}{3(N+3)}\right) \ln ^{2}\left(\frac{Q^{2}}{\mu^{2}}\right) \\
& \Delta_{g g}^{(2), C_{F} T_{f} n_{f}}=-\frac{608}{27 N}-\frac{192}{(N+1)^{2}}+\frac{4144}{9(N+1)}+\frac{128}{(N+2)^{2}}-\frac{3280}{9(N+2)} \\
& +\frac{1088}{9(N+3)^{2}}-\frac{1984}{27(N+3)}-\frac{256}{9 N^{2}}+\left(\frac{256}{3(N+3)^{2}}-\frac{1088}{9(N+3)}\right) S_{1}(N+3) \\
& +\frac{64}{3 N^{3}}-\frac{80}{(N+1)^{4}}+\frac{104}{(N+1)^{3}}-\frac{80}{(N+2)^{4}}+\frac{56}{(N+2)^{3}}-\frac{64}{(N+3)^{3}}
\end{aligned}
$$




$$
\begin{aligned}
& +32 \zeta_{3}+\frac{64 \zeta_{2}}{3 N}-32 \frac{\zeta_{2}}{(N+1)^{2}}+48 \frac{\zeta_{2}}{(N+1)}-32 \frac{\zeta_{2}}{(N+2)^{2}}-32 \frac{\zeta_{2}}{(N+2)}-\frac{64 \zeta_{2}}{3(N+3)} \\
& +\frac{64 S_{2}(N+3)}{3(N+3)}-\frac{64 S_{2}(N)}{3 N}-32 \frac{A_{18}(N+1)}{(N+1)}+16 \frac{A_{18}(N+2)}{(N+2)}+32 \frac{A_{18}(N)}{N} \\
& -\frac{128 S_{1}^{2}(N+3)}{3(N+3)}+\frac{128 S_{1}^{2}(N)}{3 N}-\frac{134}{3}+\left(-32 \frac{\zeta_{2}}{N}+32 \frac{S_{2}(N)}{N}-\frac{128}{3 N^{2}}+\frac{512}{9 N}\right) S_{1}(N) \\
& +\left(\frac{32}{3 N}+\frac{8}{(N+1)}-\frac{8}{(N+2)}-\frac{16}{(N+2)^{2}}-\frac{16}{(N+1)^{2}}-\frac{32}{3(N+3)}\right) \ln ^{2}\left(\frac{Q^{2}}{\mu^{2}}\right) \\
& +\left(-\frac{64}{(N+1)^{2}}+\frac{32}{(N+1)}\right) S_{1}^{2}(N+1)+\left(\left(\frac{64}{(N+1)^{2}}-\frac{32}{(N+1)}\right) S_{1}(N+1)\right. \\
& +\left(\frac{32}{(N+2)}+\frac{64}{(N+2)^{2}}\right) S_{1}(N+2)+8+\frac{64}{(N+1)^{2}}-\frac{256}{9 N}+\frac{128 S_{1}(N+3)}{3(N+3)} \\
& -\frac{128 S_{1}(N)}{3 N}+\frac{64}{3 N^{2}}+\frac{48}{(N+2)^{2}}-\frac{64}{(N+1)^{3}}-\frac{128}{3(N+3)^{2}}+\frac{544}{9(N+3)} \\
& \left.+\frac{352}{3(N+2)}-\frac{448}{3(N+1)}-\frac{64}{(N+2)^{3}}\right) \ln \left(\frac{Q^{2}}{\mu^{2}}\right)+\left(-\frac{64}{(N+2)^{2}}\right. \\
& \left.-\frac{32}{(N+2)}\right) S_{1}^{2}(N+2)+\left(\frac{128}{(N+1)^{3}}+\frac{896}{3(N+1)}+32 \frac{\zeta_{2}}{(N+1)}-\frac{128}{(N+1)^{2}}\right. \\
& \left.-32 \frac{S_{2}(N+1)}{(N+1)}\right) S_{1}(N+1)+\left(\frac{32}{(N+2)}+\frac{32}{(N+2)^{2}}\right) S_{2}(N+2)+\left(\frac{32}{(N+1)^{2}}\right. \\
& \left.-\frac{48}{(N+1)}\right) S_{2}(N+1)+\left(-\frac{704}{3(N+2)}+\frac{128}{(N+2)^{3}}-\frac{96}{(N+2)^{2}}-16 \frac{\zeta_{2}}{(N+2)}\right. \\
& \left.+16 \frac{S_{2}(N+2)}{(N+2)}\right) S_{1}(N+2) \\
& \Delta_{q g}^{(2), C_{F}^{2}}=-\frac{1393}{54 N}-\frac{69}{(N+1)^{2}}-\frac{130}{9(N+1)}+\frac{214}{3(N+2)^{2}}+\frac{17}{18(N+2)} \\
& -\frac{112}{9(N+3)^{2}}+\frac{1304}{27(N+3)}+\frac{106}{9 N^{2}}-\frac{20}{(N+1)^{4}}-\frac{28}{(N+1)^{3}}+\frac{10}{(N+2)^{4}} \\
& -\frac{63}{(N+2)^{3}}-\frac{80}{3(N+3)^{3}}+\frac{16 \zeta_{2}}{3 N}-16 \frac{\zeta_{3}}{N}-8 \frac{\zeta_{2}}{(N+1)^{2}}+16 \frac{\zeta_{3}}{(N+1)}+4 \frac{\zeta_{2}}{(N+2)^{2}} \\
& +6 \frac{\zeta_{2}}{(N+2)}-8 \frac{\zeta_{3}}{(N+2)}-\frac{16 \zeta_{2}}{3(N+3)}+\frac{16 S_{2}(N+3)}{3(N+3)}-\frac{40 S_{3}(N+1)}{3(N+1)}+\frac{20 S_{3}(N+2)}{3(N+2)}
\end{aligned}
$$




$$
\begin{aligned}
& +\frac{40 S_{3}(N)}{3 N}-\frac{32 \beta^{(1)}(N+1)}{3 N}-16 \frac{\beta^{(1)}(N+2)}{(N+1)}-16 \frac{\beta^{(1)}(N+3)}{(N+2)}-\frac{32 \beta^{(1)}(4+N)}{3(N+3)} \\
& +\frac{52 S_{1}^{3}(N+1)}{3(N+1)}-\frac{26 S_{1}^{3}(N+2)}{3(N+2)}-\frac{52 S_{1}^{3}(N)}{3 N}+\left(\left(\frac{32}{(N+1)^{2}}-\frac{64}{(N+1)}\right) S_{1}(N+1)\right. \\
& +\left(\frac{28}{(N+2)}-\frac{16}{(N+2)^{2}}\right) S_{1}(N+2)+\left(\frac{36}{N}-\frac{16}{N^{2}}\right) S_{1}(N)+12 \frac{S_{1}^{2}(N+2)}{(N+2)} \\
& -24 \frac{S_{1}^{2}(N+1)}{(N+1)}+\frac{106}{9 N}-4 \frac{S_{2}(N+2)}{(N+2)}-8 \frac{S_{2}(N)}{N}-\frac{12}{(N+1)}-\frac{16}{3(N+3)^{2}} \\
& +8 \frac{S_{2}(N+1)}{(N+1)}-\frac{4}{(N+2)}-\frac{38}{(N+2)^{2}}-\frac{16}{(N+1)^{3}}+\frac{8}{(N+2)^{3}}-\frac{124}{9(N+3)} \\
& \left.+24 \frac{S_{1}^{2}(N)}{N}+\frac{8}{(N+1)^{2}}\right) \ln \left(\frac{Q^{2}}{\mu^{2}}\right)+\left(\frac{32}{3(N+3)^{2}}+\frac{248}{9(N+3)}\right) S_{1}(N+3) \\
& +\left(\frac{48}{(N+1)^{3}}-\frac{56}{(N+1)^{2}}+\frac{144}{(N+1)}-12 \frac{S_{2}(N+1)}{(N+1)}\right) S_{1}(N+1)+\left(-\frac{878}{9 N}\right. \\
& \left.+\frac{36}{N^{2}}-\frac{16}{N^{3}}+12 \frac{S_{2}(N)}{N}\right) S_{1}(N)+\left(\frac{88}{(N+2)^{2}}-\frac{24}{(N+2)^{3}}+6 \frac{S_{2}(N+2)}{(N+2)}\right. \\
& \left.-\frac{48}{(N+2)}\right) S_{1}(N+2)+\left(\frac{94}{(N+1)}-\frac{40}{(N+1)^{2}}\right) S_{1}^{2}(N+1)+\left(-4 \frac{S_{1}(N+2)}{(N+2)}\right. \\
& \left.+\frac{4}{(N+1)}-8 \frac{S_{1}(N)}{N}+8 \frac{S_{1}(N+1)}{(N+1)}-\frac{4}{(N+1)^{2}}-\frac{1}{(N+2)}+\frac{2}{(N+2)^{2}}\right) \ln ^{2}\left(\frac{Q^{2}}{\mu^{2}}\right) \\
& +\left(-\frac{60}{N}+\frac{24}{N^{2}}\right) S_{1}^{2}(N)+\left(\frac{20}{(N+2)^{2}}-\frac{45}{(N+2)}\right) S_{1}^{2}(N+2)+\left(-\frac{8}{(N+2)^{2}}\right. \\
& \left.+\frac{1}{(N+2)}\right) S_{2}(N+2)+\left(\frac{16}{(N+1)^{2}}-\frac{18}{(N+1)}\right) S_{2}(N+1)+\left(\frac{20}{3 N}-\frac{8}{N^{2}}\right) S_{2}(N) \\
& \Delta_{q g}^{(2), C_{A} C_{F}}=-\frac{21539}{54 N}-\frac{2660}{9(N+1)^{2}}+\frac{9962}{27(N+1)}-\frac{1079}{9(N+2)^{2}} \\
& +\frac{1171}{54(N+2)}-\frac{224}{9(N+3)^{2}}-\frac{238}{27(N+3)}+\frac{2402}{9 N^{2}}+\frac{48}{N^{4}}-\frac{124}{N^{3}} \\
& +\frac{72}{(N+1)^{4}}+\frac{688}{3(N+1)^{3}}+\frac{60}{(N+2)^{4}}-\frac{20}{3(N+2)^{3}}+\frac{136}{3(N+3)^{3}} \\
& +104 \frac{\zeta_{2}}{N^{2}}-204 \frac{\zeta_{2}}{N}+36 \frac{\zeta_{3}}{N}+8 \frac{\zeta_{2}}{(N+1)^{2}}+\frac{628 \zeta_{2}}{3(N+1)}-36 \frac{\zeta_{3}}{(N+1)}+76 \frac{\zeta_{2}}{(N+2)^{2}}
\end{aligned}
$$




$$
\begin{aligned}
& -\frac{14 \zeta_{2}}{3(N+2)}+18 \frac{\zeta_{3}}{(N+2)}+\frac{56 \zeta_{2}}{3(N+3)}-\frac{56 S_{2}(N+3)}{3(N+3)}+\frac{16 S_{3}(N+1)}{3(N+1)} \\
& -\frac{8 S_{3}(N+2)}{3(N+2)}-16 \frac{S_{3}(N)}{3 N}-16 \frac{\beta^{(1)}(N+1)}{N^{2}}+\frac{88 \beta^{(1)}(N+1)}{3 N}-16 \frac{\beta^{(1)}(N+2)}{(N+1)^{2}} \\
& +40 \frac{\beta^{(1)}(N+2)}{(N+1)}-8 \frac{\beta^{(1)}(N+3)}{(N+2)^{2}}+12 \frac{\beta^{(1)}(N+3)}{(N+2)}+\frac{16}{3} \frac{\beta^{(1)}(4+N)}{(N+3)}+8 \frac{\beta^{(2)}(N+1)}{N} \\
& +8 \frac{\beta^{(2)}(N+2)}{(N+1)}+4 \frac{\beta^{(2)}(N+3)}{(N+2)}+8 \frac{A_{18}(N+1)}{(N+1)}-4 \frac{A_{18}(N+2)}{(N+2)}-8 \frac{A_{18}(N)}{N} \\
& +32 \frac{S_{1}^{2}(N+3)}{(N+3)}+\frac{140 S_{1}^{3}(N+1)}{3(N+1)}-\frac{70 S_{1}^{3}(N+2)}{3(N+2)}-\frac{140 S_{1}^{3}(N)}{3 N}+\left(-\frac{784}{3 N}\right. \\
& \left.+\frac{132}{N^{2}}\right) S_{1}^{2}(N)+\left(-80 \frac{\zeta_{2}}{(N+2)}+18 \frac{S_{2}(N+2)}{(N+2)}-\frac{40}{3(N+2)^{2}}-\frac{128}{(N+2)^{3}}\right. \\
& \left.+16 \frac{\beta^{(1)}(N+3)}{(N+2)}+\frac{116}{(N+2)}\right) S_{1}(N+2)+\left(-\frac{64}{(N+3)^{2}}+\frac{64}{9(N+3)}\right) S_{1}(N+3) \\
& +\left(\frac{24}{(N+2)^{2}}+\frac{24}{N^{2}}+\frac{8}{(N+3)}-\frac{4}{3(N+2)}+24 \frac{S_{1}(N+1)}{(N+1)}+\frac{24}{(N+1)^{2}}\right. \\
& \left.-24 \frac{S_{1}(N)}{N}-12 \frac{S_{1}(N+2)}{(N+2)}+\frac{188}{3(N+1)}-\frac{230}{3 N}\right) \ln ^{2}\left(\frac{Q^{2}}{\mu^{2}}\right)+\left(-\frac{4342}{9 N}\right. \\
& \left.-\frac{128}{N^{3}}-160 \frac{\zeta_{2}}{N}+\frac{692}{3 N^{2}}+32 \frac{\beta^{(1)}(N+1)}{N}+36 \frac{S_{2}(N)}{N}\right) S_{1}(N)+\left(-\frac{68}{(N+1)^{2}}\right. \\
& \left.-\frac{106}{(N+1)}\right) S_{2}(N+1)+\left(\frac{60}{(N+1)^{2}}+\frac{634}{3(N+1)}\right) S_{1}^{2}(N+1)+\left(-\frac{46}{(N+2)^{2}}\right. \\
& \left.-\frac{15}{(N+2)}\right) S_{2}(N+2)+\left(\frac{2422}{9 N}+\frac{448}{3(N+1)^{2}}-\frac{1724}{9(N+1)}-\frac{8}{3(N+2)^{2}}\right. \\
& -\frac{362}{9(N+2)}+\frac{32}{(N+3)^{2}}-\frac{32}{9(N+3)}-\frac{124}{N^{2}}+\frac{48}{N^{3}}+\frac{64}{(N+1)^{3}} \\
& +\frac{56}{(N+2)^{3}}+72 \frac{\zeta_{2}}{N}-72 \frac{\zeta_{2}}{(N+1)}+36 \frac{\zeta_{2}}{(N+2)}-32 \frac{S_{1}(N+3)}{(N+3)}+8 \frac{S_{2}(N+1)}{(N+1)} \\
& -4 \frac{S_{2}(N+2)}{(N+2)}-8 \frac{S_{2}(N)}{N}-16 \frac{\beta^{(1)}(N+1)}{N}-16 \frac{\beta^{(1)}(N+2)}{(N+1)}-8 \frac{\beta^{(1)}(N+3)}{(N+2)} \\
& -72 \frac{S_{1}^{2}(N+1)}{(N+1)}+36 \frac{S_{1}^{2}(N+2)}{(N+2)}+72 \frac{S_{1}^{2}(N)}{N}+\left(\frac{868}{3 N}-\frac{128}{N^{2}}\right) S_{1}(N)+\left(-\frac{64}{(N+1)^{2}}\right.
\end{aligned}
$$




$$
\begin{aligned}
& \left.\left.-\frac{736}{3(N+1)}\right) S_{1}(N+1)+\left(-\frac{112}{(N+2)^{2}}-\frac{40}{3(N+2)}\right) S_{1}(N+2)\right) \ln \left(\frac{Q^{2}}{\mu^{2}}\right) \\
& +\left(-\frac{44}{N^{2}}+\frac{446}{3 N}\right) S_{2}(N)+\left(160 \frac{\zeta_{2}}{(N+1)}-\frac{96}{(N+1)^{3}}-\frac{832}{3(N+1)^{2}}\right. \\
& \left.+\frac{904}{3(N+1)}+32 \frac{\beta^{(1)}(N+2)}{(N+1)}-36 \frac{S_{2}(N+1)}{(N+1)}\right) S_{1}(N+1)+\left(\frac{114}{(N+2)^{2}}\right. \\
& \left.+\frac{97}{3(N+2)}\right) S_{1}^{2}(N+2) \\
& \Delta_{q g}^{(2), C_{F} T_{f} n_{f}}=\left(-\frac{16}{3(N+1)}+\frac{16}{3 N}+\frac{8}{3(N+2)}\right) \ln ^{2}\left(\frac{Q^{2}}{\mu^{2}}\right)+\left(\frac{16}{3(N+2)^{2}}\right. \\
& -\frac{32}{3(N+1)^{2}}-\frac{16}{3} \frac{S_{1}(N+2)}{(N+2)}+\frac{304}{9(N+1)}+\frac{32}{3 N^{2}}-\frac{152}{9(N+2)}-\frac{232}{9 N} \\
& \left.-\frac{32 S_{1}(N)}{3 N}+\frac{32 S_{1}(N+1)}{3(N+1)}\right) \ln \left(\frac{Q^{2}}{\mu^{2}}\right)+\left(\frac{32}{3(N+1)^{2}}-\frac{128}{3(N+1)}\right) S_{1}(N+1) \\
& +\left(-\frac{16}{3(N+2)^{2}}+\frac{24}{(N+2)}\right) S_{1}(N+2)+\left(-\frac{32}{3 N^{2}}+\frac{104}{3 N}\right) S_{1}(N)+\frac{1060}{27 N} \\
& -\frac{232}{9 N^{2}}+\frac{32}{3 N^{3}}-\frac{1672}{27(N+1)}+\frac{32 \zeta_{2}}{3 N}-4 \frac{S_{2}(N+2)}{(N+2)}-\frac{32}{3(N+1)^{3}} \\
& +8 \frac{S_{2}(N+1)}{(N+1)}-8 \frac{S_{2}(N)}{N}-\frac{32 \zeta_{2}}{3(N+1)}+\frac{8 S_{1}^{2}(N)}{3 N}-\frac{8 S_{1}^{2}(N+1)}{3(N+1)}+\frac{4 S_{1}^{2}(N+2)}{3(N+2)} \\
& -\frac{152}{9(N+2)^{2}}+\frac{716}{27(N+2)}+\frac{16 \zeta_{2}}{3(N+2)}+\frac{16}{3(N+2)^{3}}+\frac{304}{9(N+1)^{2}} \\
& \Delta_{q_{1} q_{2}}^{(2), C_{F}^{2}}=-\frac{210}{N}-\frac{88}{(N+1)^{2}}+\frac{188}{(N+1)}-\frac{58}{(N+2)^{2}}+\frac{22}{(N+2)} \\
& +\frac{118}{N^{2}}+\frac{32}{N^{4}}-\frac{48}{N^{3}}+\frac{32}{(N+1)^{4}}+\frac{64}{(N+1)^{3}}+\frac{8}{(N+2)^{4}}+\frac{16}{(N+2)^{3}} \\
& +32 \frac{\zeta_{2}}{N^{2}}-48 \frac{\zeta_{2}}{N}+32 \frac{\zeta_{2}}{(N+1)^{2}}+32 \frac{\zeta_{2}}{(N+1)}+8 \frac{\zeta_{2}}{(N+2)^{2}}+16 \frac{\zeta_{2}}{(N+2)} \\
& +\left(-\frac{16}{(N+2)^{3}}-\frac{40}{(N+2)^{2}}+\frac{60}{(N+2)}\right) S_{1}(N+2)+\left(-\frac{204}{N}-\frac{64}{N^{3}}\right. \\
& \left.+\frac{96}{N^{2}}\right) S_{1}(N)+\left(\frac{64}{(N+1)}+\frac{64}{(N+1)^{2}}\right) S_{1}^{2}(N+1)+\left(\frac{16}{(N+2)^{2}}\right.
\end{aligned}
$$




$$
\begin{aligned}
& \left.+\frac{32}{(N+2)}\right) S_{1}^{2}(N+2)+\left(-\frac{96}{N}+\frac{64}{N^{2}}\right) S_{1}^{2}(N)+\left(\left(-\frac{64}{(N+1)^{2}}\right.\right. \\
& \left.-\frac{64}{(N+1)}\right) S_{1}(N+1)+\left(-\frac{32}{(N+2)}-\frac{16}{(N+2)^{2}}\right) S_{1}(N+2)+\left(\frac{96}{N}\right. \\
& \left.-\frac{64}{N^{2}}\right) S_{1}(N)-\frac{72}{(N+1)}+\frac{32}{(N+1)^{3}}-\frac{30}{(N+2)}+\frac{20}{(N+2)^{2}}+\frac{102}{N} \\
& \left.+\frac{8}{(N+2)^{3}}-\frac{48}{N^{2}}+\frac{32}{N^{3}}+\frac{32}{(N+1)^{2}}\right) \ln \left(\frac{Q^{2}}{\mu^{2}}\right)+\left(-\frac{32}{(N+1)}\right. \\
& \left.-\frac{32}{(N+1)^{2}}\right) S_{2}(N+1)+\left(-\frac{8}{(N+2)^{2}}-\frac{16}{(N+2)}\right) S_{2}(N+2)+\left(-\frac{32}{N^{2}}\right. \\
& \left.+\frac{48}{N}\right) S_{2}(N)+\left(\frac{16}{(N+1)}+\frac{4}{(N+2)^{2}}+\frac{16}{N^{2}}+\frac{16}{(N+1)^{2}}-\frac{24}{N}\right. \\
& \left.+\frac{8}{(N+2)}\right) \ln ^{2}\left(\frac{Q^{2}}{\mu^{2}}\right)+\left(\frac{144}{(N+1)}-\frac{64}{(N+1)^{3}}-\frac{64}{(N+1)^{2}}\right) S_{1}(N+1) \\
& \Delta_{q q}^{(2), C_{A} C_{F}^{2}}=-\frac{16 \zeta_{3}}{N}-\frac{10}{N}+\frac{16}{(N+1)^{3}}+\frac{16}{(N+1)^{2}}+16 \frac{\zeta_{3}}{(N+1)}-\frac{32}{(N+1)} \\
& +\frac{24}{(N+2)^{3}}+\frac{36}{(N+2)^{2}}-8 \frac{\zeta_{3}}{(N+2)}+\frac{42}{(N+2)}-16 \frac{S_{3}(N+1)}{(N+1)}+8 \frac{S_{3}(N+2)}{(N+2)} \\
& +16 \frac{S_{3}(N)}{N} \\
& \Delta_{q q}^{(2), C_{F}^{3}}=32 \frac{\zeta_{3}}{N}+\frac{20}{N}-\frac{32}{(N+1)^{3}}-\frac{32}{(N+1)^{2}}-32 \frac{\zeta_{3}}{(N+1)}+\frac{64}{(N+1)} \\
& -\frac{48}{(N+2)^{3}}-\frac{72}{(N+2)^{2}}+16 \frac{\zeta_{3}}{(N+2)}-\frac{84}{(N+2)}+32 \frac{S_{3}(N+1)}{(N+1)}-16 \frac{S_{3}(N+2)}{(N+2)} \\
& -32 \frac{S_{3}(N)}{N} \\
& \Delta_{q \bar{q}}^{(2), C_{A} C_{F}^{2}}=\frac{130}{N}+\frac{296}{3(N+1)^{2}}-\frac{316}{(N+1)}-\frac{500}{3(N+2)^{2}}+\frac{890}{3(N+2)} \\
& +\frac{128}{3(N+3)^{2}}-\frac{332}{3(N+3)}-\frac{88}{3 N^{2}}-\frac{80}{(N+1)^{3}}-\frac{64}{3(N+3)^{3}}-32 \frac{\zeta_{2}}{N} \\
& +96 \frac{\zeta_{2}}{(N+1)}-96 \frac{\zeta_{2}}{(N+2)}+32 \frac{\zeta_{2}}{(N+3)}-\frac{128 S_{1}(N+3)}{3(N+3)}+48 \frac{S_{2}(N+1)}{(N+1)}
\end{aligned}
$$




$$
\begin{aligned}
& -48 \frac{S_{2}(N+2)}{(N+2)}+16 \frac{S_{2}(N+3)}{(N+3)}-16 \frac{S_{2}(N)}{N}-32 \frac{\beta^{(1)}(N+2)}{(N+1)}-16 \frac{\beta^{(1)}(N+3)}{(N+2)} \\
& -\frac{32 \beta^{(1)}(4+N)}{3(N+3)}-8 \frac{\beta^{(2)}(N+1)}{N}-8 \frac{\beta^{(2)}(N+2)}{(N+1)}-4 \frac{\beta^{(2)}(N+3)}{(N+2)}+32 \frac{A_{3}(N+1)}{(N+1)} \\
& +16 \frac{A_{3}(N+2)}{(N+2)}+32 \frac{A_{3}(N)}{N}-32 \frac{S_{1}^{2}(N+1)}{(N+1)}+32 \frac{S_{1}^{2}(N+2)}{(N+2)}-\frac{32 S_{1}^{2}(N+3)}{3(N+3)} \\
& +\frac{32 S_{1}^{2}(N)}{3 N}-32 \frac{\beta(N+1) \zeta_{2}}{N}-32 \frac{\beta(N+2) \zeta_{2}}{(N+1)}-16 \frac{\beta(N+3) \zeta_{2}}{(N+2)}+\left(\frac{128}{(N+2)}\right. \\
& \left.-16 \frac{\beta^{(1)}(N+3)}{(N+2)}\right) S_{1}(N+2)+\left(\frac{128}{3} \frac{1}{N}-32 \frac{\beta^{(1)}(N+1)}{N}\right) S_{1}(N)+\left(-\frac{88}{(N+2)}\right. \\
& \left.+\frac{88}{3(N+3)}-\frac{88}{3 N}+\frac{88}{(N+1)}\right) \ln \left(\frac{Q^{2}}{\mu^{2}}\right)+\left(-32 \frac{\beta^{(1)}(N+2)}{(N+1)}\right. \\
& \left.-\frac{128}{(N+1)}\right) S_{1}(N+1) \\
& \Delta_{q \bar{q}}^{(2), C_{F}^{3}}=-\frac{164}{3 N}+\frac{256}{3(N+1)^{2}}-\frac{64}{(N+1)}+\frac{200}{3(N+2)^{2}}+\frac{260}{3(N+2)} \\
& +\frac{32}{(N+3)^{2}}+\frac{32}{(N+3)}+\frac{80}{(N+1)^{3}}+\frac{96}{(N+2)^{3}}-\frac{32}{3(N+3)^{3}}+\frac{64 \zeta_{2}}{N} \\
& -\frac{192 \zeta_{2}}{(N+1)}+\frac{192 \zeta_{2}}{(N+2)}-\frac{64 \zeta_{2}}{(N+3)}-\frac{64 S_{2}(N+1)}{(N+1)}+64 \frac{S_{2}(N+2)}{(N+2)} \\
& -\frac{64 S_{2}(N+3)}{3(N+3)}+\frac{64 S_{2}(N)}{3 N}+64 \frac{\beta^{(1)}(N+2)}{(N+1)}+32 \frac{\beta^{(1)}(N+3)}{(N+2)}+16 \frac{\beta^{(2)}(N+1)}{N} \\
& +16 \frac{\beta^{(2)}(N+2)}{(N+1)}+8 \frac{\beta^{(2)}(N+3)}{(N+2)}-64 \frac{A_{3}(N+1)}{(N+1)}-32 \frac{A_{3}(N+2)}{(N+2)}-64 \frac{A_{3}(N)}{N} \\
& -32 \frac{S_{1}^{2}(N+1)}{(N+1)}+32 \frac{S_{1}^{2}(N+2)}{(N+2)}-\frac{32 S_{1}^{2}(N+3)}{3(N+3)}+\frac{32 S_{1}^{2}(N)}{3 N}+64 \frac{\beta(N+1) \zeta_{2}}{N} \\
& +64 \frac{\beta(N+2) \zeta_{2}}{(N+1)}+32 \frac{\beta(N+3) \zeta_{2}}{(N+2)}+\left(-\frac{64}{3(N+2)}+64 \frac{S_{1}(N+1)}{(N+1)}-\frac{64 S_{1}(N)}{3 N}\right. \\
& -64 \frac{S_{1}(N+2)}{(N+2)}+\frac{64}{3(N+1)}+\frac{32}{(N+2)^{2}}-\frac{64}{3(N+3)^{2}}-\frac{32}{(N+1)^{2}} \\
& \left.+\frac{64 S_{1}(N+3)}{3(N+3)}\right) \ln \left(\frac{Q^{2}}{\mu^{2}}\right)+\left(64 \frac{\beta^{(1)}(N+2)}{(N+1)}+\frac{96}{(N+1)^{2}}-\frac{352}{3(N+1)}\right) S_{1}(N+1)
\end{aligned}
$$




$$
\begin{aligned}
& +\left(\frac{352}{3(N+2)}-\frac{96}{(N+2)^{2}}+32 \frac{\beta^{(1)}(N+3)}{(N+2)}\right) S_{1}(N+2)+\left(-\frac{32}{(N+3)}\right. \\
& \left.+\frac{128}{3(N+3)^{2}}\right) S_{1}(N+3)+\left(\frac{32}{N}-\frac{64}{3 N^{2}}+64 \frac{\beta^{(1)}(N+1)}{N}\right) S_{1}(N) \\
& \Delta_{q \bar{q}}^{(2), C_{F}^{2} T_{f} n_{f}}=\left(-\frac{32}{3(N+3)}-\frac{32}{(N+1)}+\frac{32}{3 N}+\frac{32}{(N+2)}\right) \ln \left(\frac{Q^{2}}{\mu^{2}}\right)+\frac{32}{3 N^{2}} \\
& +\frac{64 S_{1}(N+3)}{9(N+3)}-\frac{368}{27 N}-\frac{160}{3(N+1)^{2}}-\frac{688}{9(N+2)}+\frac{592}{9(N+1)}+\frac{128}{3(N+2)^{2}} \\
& +\frac{656}{27(N+3)}+\frac{64 S_{1}(N+1)}{3(N+1)}-\frac{128}{9(N+3)^{2}}-\frac{64 S_{1}(N)}{9 N}-\frac{64 S_{1}(N+2)}{3(N+2)} .
\end{aligned}
$$




\section{A.4 Pseudo-scalar Higgs Coefficient Functions}

In this appendix we present the Mellin transforms of the coefficient functions of the hadronic pseudo-scalar Higgs-boson cross section in the heavy-mass limit. We used the same notation as in Refs. [6].

$$
\begin{aligned}
& \Delta_{a b, A-H}=\Delta_{a b, A-H}^{(0)}+a_{s} \Delta_{a b, A-H}^{(1)}+a_{s}^{2} \Delta_{a b, A-H}^{(2)} \\
& \Delta_{g g, A-H}^{(0)}=0 \\
& \Delta_{g g, A-H}^{(1)}=+8 C_{A} \\
& \Delta_{g q, A-H}^{(1)}=0 \\
& \Delta_{q \bar{q}, A-H}^{(1)}=0 \\
& \Delta_{g g, A-H}^{(2), C_{A}^{2}}=\left(-\frac{20}{3}-\frac{64}{(N+3)}+\frac{64}{(N+2)}-\frac{128}{(N+1)}-64 S_{1}(N-1)\right) \ln \left(\frac{Q^{2}}{\mu^{2}}\right) \\
& +\frac{215}{3}-\frac{32}{N^{2}}+\frac{44}{3 N}-\frac{32}{(N+1)^{3}}-\frac{440}{3(N+1)^{2}}+128 \frac{S_{1}(N+3)}{(N+3)}+\frac{232}{3(N+1)} \\
& +\frac{64}{(N+2)^{2}}+\frac{176}{3(N+3)}-\frac{452}{3(N+2)}-\frac{64}{(N+3)^{2}}+64 S_{1}^{2}(N-1) \\
& +256 \frac{S_{1}(N+1)}{(N+1)}+128 \zeta_{2}-128 \frac{S_{1}(N+2)}{(N+2)} \\
& \Delta_{g g, A-H}^{(2), C_{A} T_{f} n_{f}}=-\frac{4}{3}+\frac{16}{3 N}-\frac{32}{3(N+1)^{2}}+\frac{16}{3(N+1)}-\frac{32}{3(N+2)} \\
& +\frac{32}{3} O_{2} L_{t}-\frac{16}{3} O_{2}-\frac{8}{3} \ln \left(\frac{Q^{2}}{\mu^{2}}\right) \\
& \Delta_{g g, A-H}^{(2), C_{F} T_{f} n_{f}}=-50-\frac{16}{N}+\frac{32}{(N+1)^{3}}+\frac{32}{(N+1)}-\frac{16}{(N+2)}-8 \ln \left(\frac{Q^{2}}{\mu^{2}}\right) \\
& \Delta_{q g, A-H}^{(2), C_{F}^{2}}=-12 \frac{O_{2}}{N}-\frac{4}{N}+\frac{16}{(N+1)^{3}}+\frac{24}{(N+1)^{2}}+\frac{16}{(N+1)}+12 \frac{O_{2}}{(N+2)} \\
& -\frac{12}{(N+2)} \\
& \Delta_{q g, A-H}^{(2), C_{A} C_{F}}=\left(\frac{32}{N}-\frac{32}{(N+1)}+\frac{16}{(N+2)}\right) \ln \left(\frac{Q^{2}}{\mu^{2}}\right)+\frac{4}{(N+2)}+\frac{60}{N}
\end{aligned}
$$




$$
\begin{aligned}
& -\frac{32}{(N+1)^{3}}-\frac{80}{(N+1)^{2}}-\frac{48}{(N+1)}+\frac{16}{(N+2)^{2}}-64 \frac{S_{1}(N)}{N}+64 \frac{S_{1}(N+1)}{(N+1)} \\
& -32 \frac{S_{1}(N+2)}{(N+2)} \\
& \Delta_{q g, A-H}^{(2), C_{F} T_{f} n_{f}}=0 \\
& \Delta_{q_{1} q_{2}, A-H}^{(2), C_{F}^{2}}=-\frac{32}{N^{2}}+\frac{88}{N}-\frac{32}{(N+1)^{3}}-\frac{48}{(N+1)^{2}}-\frac{96}{(N+1)}+\frac{8}{(N+2)} \\
& \Delta_{q q, A-H}^{(2), C_{A} C_{F}^{2}}=\frac{32}{N}-\frac{32}{(N+1)^{3}}-\frac{32}{(N+1)^{2}}-\frac{32}{(N+1)} \\
& \Delta_{q q, A-H}^{(2), C_{F}^{3}}=-\frac{64}{N}+\frac{64}{(N+1)^{3}}+\frac{64}{(N+1)^{2}}+\frac{64}{(N+1)} \\
& \Delta_{q \bar{q}, A-H}^{(2), C_{A} C_{F}^{2}}=\frac{8}{3 N}+\frac{32}{(N+1)^{3}}-\frac{80}{3(N+1)^{2}}+\frac{56}{3(N+2)}-\frac{64}{3(N+3)} \\
& \Delta_{q \bar{q}, A-H}^{(2), C_{F}^{3}}=-48 \frac{O_{2}}{N}+\frac{64}{N}-\frac{32}{(N+1)^{3}}+\frac{32}{(N+1)^{2}}+96 \frac{O_{2}}{(N+1)}-\frac{160}{(N+1)} \\
& -48 \frac{O_{2}}{(N+2)}+\frac{96}{(N+2)} \\
& \Delta_{q \bar{q}, A-H}^{(2), C_{F}^{2} T_{f} n_{f}}=-\frac{32}{3 N}+\frac{64}{3(N+1)^{2}}+\frac{32}{3(N+2)},
\end{aligned}
$$

where

$$
O_{2}=1 \quad L_{t}=\ln \left(\frac{\mu_{R}^{2}}{m_{t}^{2}}\right)
$$




\section{References}

[1] William of Occam, Quadlibeta, Book V, (1324).

[2] R.K. Ellis, I. Hinchliffe, M. Soldate, J.J. van der Bij, Nucl. Phys. B297 (1988) 221;

U. Baur, E. Glover, Nucl. Phys. B339 (1990) 38;

D. Graudenz, M. Spira, P. Zerwas, Phys. Rev. Lett. 70 (1993) 1372;

M. Spira, A. Djouadi, D. Graudenz, P. Zerwas, Phys. Lett. B318 (1993) 347; Nucl. Phys. B453 (1995) 17.

[3] A. Djouadi, hep-ph/0503172, hep-ph/0503173.

[4] S. Dawson, Nucl. Phys. B359 (1991) 283; A. Djouadi, M. Spira, P. Zerwas, Phys. Lett. B264 (1991) 440

[5] T. Matsuura, S.C. van der Marck, and W.L. van Neerven, Nucl. Phys. B319 (1989) 570;

R. Hamberg, W.L. van Neerven, and T. Matsuura, Nucl. Phys. B359 (1991) 343;

V. Ravindran, J. Smith and W. L. van Neerven, Nucl. Phys. B682 (2004) 421.

[6] S. Catani, D. de Florian, and M. Grazzini, JHEP (2001) 0105:025;

R.V. Harlander, W.B. Kilgore, Phys. Rev. D64 (2001) 013015; Phys. Rev. Lett. 88 (2002) 201801; JHEP (2002) 0210:017;

C. Anastasiou and K. Melnikov, Nucl. Phys. B646 (2002) 220;

V. Ravindran, J. Smith, and W. L. van Neerven, Nucl. Phys. B665 (2003) 325; B704 (2005) 332 .

J. Smith and W.L. van Neerven, hep-ph/0501098;

C. Anastasiou, K. Melnikov, and F. Petriello, hep-ph/0501130.

[7] J. Blümlein and S. Kurth, Phys. Rev. D60 (1999) 014018.

[8] Tevatron Electroweak Working Group, D0 collab., hep-ex/0404010.

[9] CMS collab., Technical Proposal, report CERN/LHCC/94-38, ATLAS Coll., ATLAS Detector and Physics Performance: Technical Design Report, Vol. 2, report CERN/LHCC/99-15 (1999).

[10] S. Moch, J.A.M. Vermaseren, and A. Vogt, Nucl. Phys. B688 (2004) 101; B691 (2004) 129.

[11] N. Nielsen, Nova Acta Leopold. XC (1909) 121;

S. Kölbig, Siam J. Math. Anal. 17 (1986) 1232;

L. Lewin, Dilogarithms and Associated Functions (Macdonald, London, 1958);

Polylogarithms and Associated Functions (North Holland, New York, 1981).

[12] J. Blümlein, Comput. Phys. Commun. 133 (2000) 76.

[13] J. Blümlein and S.-O. Moch, hep-ph/0503188.

[14] J.A.M. Vermaseren, Int. J. Mod. Phys. A14 (1999) 2037.

[15] L. Euler, Novi Comm. Acad. Sci Petropolitanae 1 (1775) 140;

R.L. Graham, D.E. Knuth, and O. Patashnik, Concrete Mathematics, (Addison-Wesley, Reading/MA, 1994). 
[16] J.M. Borwein and R. Girgensohn, Electron. J. Combinatorics 3 (1996) R23 (Appendix by D.J. Broadhurst).

[17] J. Blümlein, Comput. Phys. Commun. 159 (2004) 19.

[18] J. Blümlein, Nucl. Phys. B Proc. Suppl. 135 (2004) 225, hep-ph/0407044; DESY 04-064.

[19] J. Blümlein and V. Ravindran, Nucl. Phys. B Proc. Suppl. 135 (2004) 24, hep-ph/0407045.

[20] J. Blümlein and S. Moch, DESY 05-008.

[21] J. Blümlein and V. Ravindran, in preparation.

[22] J. Blümlein and A. Vogt, Phys. Lett. B370 (1996) 149; B 386 (1996) 350; Acta Phys. Pol. B 27 (1996) 1309; Phys. Rev. D 57 (1998) 1; D58 (1998) 014020;

J. Blümlein, V. Ravindran, W.L. van Neerven and A. Vogt, hep-ph/9806368;

R.K. Ellis, Z. Kunszt, and E. Levin, Nucl. Phys. B420 (1994) 517; E : B433 (1995) 498(E).

[23] S. Alekhin and J. Blümlein, Phys. Lett. B594 (2004) 299.

[24] H. Mellin, Acta Math. 25 (1902) 139.

[25] J. Blümlein and H. Kawamura, hep-ph/0409289 and in preparation.

[26] M. Krämer, E. Laenen, M. Spira, Nucl. Phys. B511 (1998) 523;

K.G. Chetyrkin, B.A. Kniehl, and M. Steinhauser, Phys. Rev. Lett. 79 (1997) 353;

K.G. Chetyrkin, B.A. Kniehl, M. Steinhauser, and W.A. Bardeen, Nucl. Phys. B535 (1998) 3.

[27] N. Nielsen, Handbuch der Theorie der Gammafunktion, (Teubner, Leipzig, 1906).

[28] E. Carlson, Thesis, Univ. Uppsala, 1914, E.C. Titchmarsh, Theory of Functions, (Oxford University Press, Oxford, 1939), Chap.9.5. 